\title{
Valence bond approach and Verma bases
}

\author{
Josef Paldus · Josep Planelles
}

(Received

/ Accepted

J. Paldus $(\otimes)$

Department of Applied Mathematics, University of Waterloo, Waterloo, Ontario, Canada N2L 3G1 e-mail: paldus@uwaterloo.ca

J. Planelles

Dept. de Química Física i Analítica, Universitat Jaume I, 12080, Castelló, Spain e-mail: josep.planelles@uji.es 


\begin{abstract}
The unitary group approach (UGA) to the many-fermion problem is based on the Gel'fand-Tsetlin (G-T) representation theory of the unitary or general linear groups. It exploits the group chain $\mathrm{U}(n) \supset \mathrm{U}(n-1) \supset \cdots \supset \mathrm{U}(2) \supset \mathrm{U}(1)$ and the associated G-T triangular tableau labeling basis vectors of the relevant irreducible representations (irreps). The general G-T formalism can be drastically simplified in the many-electron case enabling an efficient exploitation in either configuration interaction (CI) or coupled cluster (CC) approaches to the molecular electronic structure. However, while the reliance on the G-T chain provides an excellent general formalism from the mathematical point of view, it has no specific physical significance and dictates a fixed Yamanouchi-Kotani coupling scheme, which in turn leads to a rather arbitrary linear combination of distinct components of the same multiplet with a given orbital occupancy. While this is of a minor importance in molecular orbital (MO) based CI approaches, it is very inconvenient when relying on the valence bond (VB) scheme, since the G-T states do not correspond to canonical Rumer structures. While this shortcoming can be avoided by relying on the Clifford algebra UGA (CAUGA) formalism, which enables an exploitation of a more or less arbitrary coupling scheme, it is worthwhile to point out the suitability of the so-called Verma basis sets for the VB-type approaches.
\end{abstract}

Keywords Valence bond (VB) method • unitary group approach (UGA) . Verma bases $\cdot$ covalent and ionic VB structures $\cdot \pi$-electron model $\cdot$ canonical vs non-canonical VB structures 


\section{Introduction}

The unitary group approach (UGA) to the many-electron correlation problem proved to be very useful in key approaches to the molecular electronic structure, irrespective whether based on the configuration interaction (CI) or coupled cluster (CC) methodologies (see, e.g., [1-22]). In each case one relies on the molecular orbital (MO) formalism rather than on the atomic orbital (AO) based valence bond (VB) method in spite of the fact that the latter are better disposed to a chemical intuition and interpretation. Needless to say, however, that the nonorthogonality of the AOs leads to the well-known $N$ ! problem which greatly complicates VB approaches, particularly when applied in an $a b$ initio context. For this very reason one often relies on an orthonormalized form of the AOs in which case one employs the acronym $\overline{\mathrm{VB}}[23]$ (see also the generalized VB (GVB) [24], spin-coupled VB [25], and PPP-VB [26] approaches). The same applies to semi-empirical approaches to $\pi$-electron systems with conjugated double bonds of either the Hückel or the Pariser-Parr-Pople (PPP) type, in which case one relies on hypothetical Löwdin or symmetrically orthonormalized AOs.

An exploitation of a representation theory of the unitary group $\mathrm{U}(n)$ - or rather of its Lie algebra ${ }^{1}(\mathrm{LA}) \mathfrak{u}(n)$ - when handling many-fermion systems stems from the fact that the second quantized version of the relevant Hamiltonian $H$ can be express as a second degree polynomial in terms of the $\mathrm{U}(\infty)$ generators. Although this observation was made already in 1935 by Jordan [27], the relevant formalism for an actual exploitation - both in the algebra and the quantum chemical methodology - was not available until much later.

Now, in the MO based methods one exploits a finite dimensional $N$-electron subspace of a relevant Fock space spanned by antisymmetrized products of $2 n$ orthonormal molecular spinorbitals, which in turn are expressed as linear combinations of

\footnotetext{
${ }^{1}$ Needless to say that in view of a close relationship between the Lie groups and their Lie algebras one often does not distinguish between these object, especially in physics literature when employing a relevant representation theory.
} 
atomic (spin)orbitals (LCAO approximation). When modeling molecular systems one usually relies on a spin-independent electronic Hamiltonian $H$ that can be expressed as a second degree polynomial in terms of $\mathrm{U}(n)$ generators and on a non-relativistic, time-independent Schrödinger equation. When the spin effects play an important, though subsidiary, role, as when dealing with phenomena such as the intersystem crossing, phosphorescent lifetimes, molecular predissociation, etc., or when interpreting high-resolution spectra, the spin-independent, non-relativistic wave functions still provide an excellent starting point for the description of such phenomena. Even here UGA provides an efficient tool (see, e.g., [28] and references therein).

Thus, in MO based approaches the relevant LAs are the spinorbital LA $\mathfrak{u}(2 n)$ and the orbital LA $\mathfrak{u}(n)$, spanned by the generators $E_{A A^{\prime}} \equiv E_{a \sigma, a^{\prime} \sigma^{\prime}}$ and $E_{a a^{\prime}}$, respectively, the latter representing partial traces over the spin variables $\sigma$. The spinorbitals are then represented via a simple product of the orbital $(|a\rangle)$ and spin $(|\sigma\rangle)$ components, i.e., $|A\rangle=|a\rangle|\sigma\rangle$. Thus $E_{a a^{\prime}}=\sum_{\sigma} E_{a \sigma, a^{\prime} \sigma}$, where $E_{a \sigma, a^{\prime} \sigma^{\prime}} \equiv E_{A A^{\prime}}=X_{A}^{\dagger} X_{A^{\prime}}$, with $X_{A}^{\dagger}$ and $X_{A}$ representing the spinorbital creation and annihilation operators (see, e.g., $[1,3,6,10,13]$ for details). These generators then satisfy the usual $\mathfrak{u}(n)$ [or, in fact $\mathfrak{g l}(n)]$ commutation relations, e.g., $\left[E_{a b}, E_{c d}\right]=\delta_{b c} E_{a d}-\delta_{a d} E_{c b}$, and similarly for the spinorbital generators $E_{A A^{\prime}}$.

The relevant $\mathrm{U}(n)$ or $\mathrm{GL}(n)$ representation theory was developed in the fifties by Gel'fand and Tsetlin [29] (G-T), who exploited the fact that the $\mathrm{U}(n) \downarrow \mathrm{U}(n-1) \otimes \mathrm{U}(1)$ subduction is multiplicity free, as was pointed out already by Weyl in the second edition of his well known monograph [30] (allegedly this was known already to I. Schur [31]). The irreducible representations (irreps) $\Lambda$ of $\mathrm{U}(n)$ or $\operatorname{GL}(n)$ are uniquely labeled by a non-increasing sequence of integers $\left\langle\lambda_{1}, \lambda_{2}, \cdots, \lambda_{n}\right\rangle$, representing the highest weight (cf. Appendix). Labeling similarly highest weights of the subduced $\mathrm{U}(n-1)$ subalgebra irreps by $\left\langle\mu_{1}, \mu_{2}, \cdots, \mu_{n-1}\right\rangle$, the pertinent $\mathrm{U}(n-1)$ irrep weights are given by the so-called betweenness conditions $\lambda_{i} \leqslant \mu_{i} \leqslant \lambda_{i+1},(i=1, \cdots, n-1)$. 
Gel'fand and Tsetlin then employed the subgroup chain

$$
\mathrm{U}(n) \supset \mathrm{U}(n-1) \supset \cdots \supset \mathrm{U}(1)
$$

arranging weights for the subsequent subalgebras into a triangular pattern, satisfying the betweenness conditions at each level. These patterns or tableaux then uniquely label the basis vectors of a given $\mathrm{U}(n)$ irrep $\Lambda$ in view of the fact that $\mathrm{U}(1)$ is abelian. Moreover, G-T also presented explicit formulas for the matrix elements of $\mathfrak{u}(n)$ generators in this basis.

Now, in the spin-independent case, one can achieve a spin-free, automatically spin-adapted formalism, by relying on a subgroup $\mathrm{U}(n)$ of the spin-orbital group $\mathrm{U}(2 n)$ by exploiting the chain $\mathrm{U}(2 n) \supset \mathrm{U}(n) \otimes \mathrm{SU}(2)$. Indeed, considering $n$ MOs occupied by $N$ electrons one easily finds a unique irrep of $\mathrm{U}(n)$ for any spin multiplicity $2 S+1, S$ being the total spin quantum number. In order to yield a physically relevant, totally antisymmetric irrep of $\mathrm{U}(2 n)$, the $\mathrm{U}(n)$ irrep must be conjugate to that for $\mathrm{SU}(2)$, implying at most two-column $\mathrm{U}(n)$ irreps $\Gamma$ of the form $\langle 2,2, \cdots, 2,1,1, \cdots, 1,0,0, \cdots, 0\rangle \equiv\left\langle 2^{a} 1^{b} 0^{c}\right\rangle, \Gamma \equiv \Gamma(a, b, c)$, with $a, b$, and $c$ indicating number of 2's, 1's, and 0's, respectively [1]. One easily finds that the three parameters $a, b$, and $c$ are uniquely determined by the number of MOs $n$, the number of electrons $N$, and the total spin $S$, namely $a=N / 2-S, b=2 S$ and $c=n-M / 2-S$.

In principle, one can chose any basis for the $\mathrm{U}(n)$ irrep $\Gamma(a, b, c) \equiv\left\langle 2^{a} 1^{b} 0^{c}\right\rangle$, the basis elements of which represent spin-adapted configuration state functions (CSFs) associated with a chosen spin-coupling scheme. In the G-T case, this basis is orthonormal and corresponds to the Yamanouchi-Kotani coupling scheme. Although the G-T basis may not be the most appropriate one from the chemical viewpoint (see, e.g., $[32,33]$ ), its great advantage is the availability of the explicit expressions for the generator matrix elements. While in the general case the latter are rather unwieldy and the G-T patterns involve $n(n+1) / 2$ parameters, the entire formalism can be drastically simplified when dealing with the two-column $\Gamma(a, b, c)$ irreps that are relevant when studying many-electron systems [1]. Moreover, in view of the as- 
sociation with the $\mathfrak{s u}(2)$ LA, there is a possibility to exploit graphical methods of spin algebras providing another efficient route to the evaluation of required matrix elements of generators and products of generators [34].

Indeed, the basis vectors (or CSFs) of a carrier space of the irrep $\Gamma(a, b, c)$ of dimension

$$
\operatorname{dim} \Gamma(a, b, c)=\frac{b+1}{n+1}\left(\begin{array}{c}
n+1 \\
a
\end{array}\right)\left(\begin{array}{c}
n+1 \\
c
\end{array}\right)
$$

may be labeled by the $n \times 3$ ABC tableau [P] [1,3,10] (often called Paldus or Gel'fandPaldus or electronic $G$-T tableau, see, e.g., $[4,5,8,9,35-42])$. The rows of $[P] \equiv$ $\left\|a_{i} b_{i} c_{i}\right\|_{n \times 3}$, i.e., $\left(a_{i}, b_{i}, c_{i}\right), a_{i}+b_{i}+c_{i}=i$, label the irreps of the LAs of $\mathrm{U}(i)$ in the canonical chain (1). Even more efficiently one can employ the labeling by a twocolumn $\Delta a \overline{\Delta c}$ tableaux, where $\Delta x_{i}=x_{i}-x_{i-1}$ and $\overline{\Delta x_{i}}=1-\Delta x_{i}(i=0,1, \cdots, n)$, with $x_{n} \equiv x, x_{0} \equiv 0, x=a, b$, and $c$. Clearly, $\Delta a_{i}+\Delta b_{i}+\Delta c_{i}=1$. Another convenient labeling uses the ternary step numbers $d_{i}, 0 \leqslant d_{i} \leqslant 3$ (see, e.g., $[1,2,4,5,10,11,34]$ )

$$
d_{i}=1-2 \Delta a_{i}-\Delta c_{i}=2 \Delta a_{i}+\overline{\Delta c_{i}} .
$$

The general $\mathrm{U}(i) \supset \mathrm{U}(i-1)$ step involves at most four subreps characterized by these step numbers. Thus, an electronic G-T basis can also be labeled by $n$-component $d$-vectors $d=\left\{d_{i}\right\}$.

The entire electronic G-T basis may be conveniently represented by a Shavitt graph $[4,5,7,11]$ providing a compact and transparent rendering of it structure. These two-rooted graphs are the basis of the so-called graphical UGA (GUGA), which is very helpful in designing various computational strategies, particularly in an often required truncation of the full CI (FCI) basis due to its huge dimensionality, as well as in graphical visualization of a segmentation of the generator matrix elements. Recently, the GUGA representation of the electronic G-T basis was exploited by Shepard and collaborators [43-45]. Their approach makes it possible to handle extremely large CI expansions, exceeding traditional ones by several orders of magnitude .

A very succinct early presentation of an explicit construction of the GL( $n)$ and 
$\mathrm{O}(n)$ irreps by G-T [29] was followed by an important independent work providing detailed proofs and insights, resulting in an extensive development of the subject. A comprehensive derivation of G-T results and a formulation of the related tensor operator formalism was given by Baird and Biedenharn $[46,47]$ and the lowering operator approach was advanced by Nagel and Moshinsky [48,49], as well as by Zhelobenko [50,51] and Hou Pei-yu [52]. A completely independent approach based on polynomial identities for generators and implied projection operators was then developed by Green [53] and Gould [54-57]. The latter author and collaborators also applied these techniques in the framework of quantum chemical UGA formalism [38-42].

Here we should mention that the evaluation of $\mathrm{U}(n)$ generator matrix elements in the context of many-Fermion systems can be often facilitated by considering its relationship with the symmetric or permutation group $S_{n}$, as well as with the spinangular-momentum group $\mathrm{SU}(2)$. In the latter case it is especially rewarding to exploit graphical method of spin algebras [58-62], which led to an efficient evaluation of matrix elements of generator products that are required in handling of the twobody part of the electronic Hamiltonian. Yet another useful approach has been based on spin-adapted creation and annihilation operators [63-65].

At this point we should also recollect other than CI exploitations of UGA formalism, namely its employment: (i) in the MC-SCF method [66-69], (ii) in the many-body perturbation theory (MBPT) [70], (iii) in CC methods [14-22, 71-79], (iv) in quantum dots [80], (v) in system partitioning of composite systems or (vi) in CAS-CI [65, 81-86], (vii) in VB approaches [87-94], (viii) in reduced density matrices (RDMs) [95,96], (ix) in nuclear magnetic resonance spectra (NMR) [97], or (x) in charge migration in fragmentation of peptide ions $[98,99]$. We also mention further innovations and extensions, such as bonded tableaux UGA [100] and exploitation of parastatistics and para-Fermi algebras $[101,102]$. Finally, as already pointed out above, UGA also provides a good starting point for the handling of spin-effects in 
which case we require matrix elements of $\mathrm{U}(2 n)$ generators. Much work has been done in this direction and is still going on [28, 40,41, 103-113].

All of the above listed developments are based on the G-T type canonical bases representing CSFs. As already mentioned, and as discussed in some detail in [32] (see also [33]), the G-T chain is rather artificial from the viewpoint of the molecular electronic structure applications. This also applies to the so-called generator states $[9,114-116]$ which are, moreover, nonorthogonal and over-complete, thus requiring a special attention (such as Gram-Schmidt or Löwdin orthogonalization so that the resulting states are no longer true generator states). In fact, in actual applications these were employed only for very small systems (mostly 2 - 4 electrons), in which case the required matrix elements were calculated by a brute force relying on commutation relations. Likewise, the general purpose approaches based on projection operators or the so-called crystal bases are related to the G-T chain and have a number of drawbacks (see, e.g., [33]).

A step forward in this direction was the development of the Clifford algebra UGA (CAUGA) $[32,83,101,117,118]$ that is based on the work of Sarma and collaborators $[119,120]$. Here, in lieu of G-T chain one exploits the imbedding of $\mathrm{U}(n)$ in a much larger group $\mathrm{U}\left(2^{n}\right)$ via the special orthogonal group $\mathrm{SO}(m), m=2 n$ or $m=2 n+1$ (i.e., the classical LAs $B_{n}$ and $\left.D_{n}\right)$ and their covering group $\operatorname{Spin}(m)$, i.e., the group chain

$$
\mathrm{U}\left(2^{n}\right) \supset \mathrm{Spin}(m) \supset \mathrm{SO}(m) \supset \mathrm{U}(n), \quad m=2 n \quad \text { and } \quad m=2 n+1,
$$

supplemented, if desired, by the G-T chain or other $\mathrm{U}(n)$ chains. As is customary in physics, we talk in terms of various groups while in fact exploiting their LAs. At the same time, one also exploits the imbedding of $\mathrm{SO}(m)$ in the $2^{n}$-dimensional Clifford algebra $C_{n}$. For the two-column irreps of $\mathrm{U}(n)$ this enabled us to rely only on two-box irreps of $\mathrm{U}\left(2^{n}\right)$. Providing an explicit representation of $\mathrm{U}(n)$ generators in terms of those for $\mathrm{U}\left(2^{n}\right)$, the evaluation of $\mathrm{U}(n)$ generator matrix elements becames a trivial counting problem [32]. This procedure enables the use of an arbitrary coupling 
scheme, including non-orthogonal Weyl states or canonical Rumer states. In fact, it makes it possible, if desired, to handle particle non-conserving processes by exploiting the $\mathrm{SO}(m)$ generators.

In this paper we wish to explore the so-called Verma bases and their relationship to the VB-type approaches. These bases have certain desirable properties both from the viewpoint of the LA representation theory and their possible exploitation in theoretical chemistry, as will be pointed out in the following Sections. They represent the so-called monomial bases defined by products of elementary lowering generators acting on the highest weight vector or state (HWS) of a given irrep. The initial idea stems from the work of Verma [121]. The first explicit formulation of such bases for simple LAs of type $A_{n}(n \geqslant 1), B_{n}$ and $C_{n}(2 \leqslant n \leqslant 6), D_{n}(4 \leqslant n \leqslant 6)$, and $G_{2}$ was given by Patera [122] and Li et al. [123]. Those for the $A_{n} \equiv \mathfrak{s l}(n+1, \mathbb{C})$, which are of our concern here, were then reconsidered by Raghavan and Sankaran [124], focusing on the proof of the linear independence of these basis sets. An entirely different approach relying on a restriction of the Poincaré-Birkhoff-Witt (PBW) bases for universal enveloping algebras (UEAs) $U(\mathfrak{g})$ of a given LA $\mathfrak{g}$ to the pertinent irreps of $\mathfrak{g}$ was employed by Littelmann [125]. The relationship of Littelmann's formulation (using an alternative reverse ordering of generators) with that of Patera et al. [122,123], while providing a simplified derivation of these bases, was recently given by Pošta and Havlíček [126].

We summarize the relevant results for the $A_{n} \equiv \mathfrak{s l}(n+1, \mathbb{C})$ LAs in the next Sect. 2 and point out the usefulness of these basis sets for the VB-type approaches in Sect. 3. We briefly discuss these results in Sect. 4 and draw the main conclusions in Sec. 5. Some useful LA concepts that are used in the paper are summarized in the Appendix. 


\section{Verma bases}

The Verma monomial basis sets for the $A_{n} \equiv \mathfrak{s l}(n+1, \mathbb{C}),(n \geqslant 1)$ LA finitedimensional irreps are determined by a set of inequalities for parameters defining ordered products of elementary lowering generators acting on the highest weight vector or HWS of a given irrep. These basis sets have a number of desirable properties, namely: (i) Their basis vectors are eigenvectors of the Cartan subalgebra and thus suitable for various modifications, since they are not related to any fixed subalgebra - as is the case, for example, for the G-T bases that are adapted to the subgroup chain (1) - and may thus be adapted to subalgebra(s) that are pertinent to a given problem. (ii) They are labeled by the 'additive quantum numbers' representing components of the weight of a given irrep (see [123]). (iii) The set of defining inequalities (sometimes referred to as Verma inequalities [126]) applies to any finite-dimensional irrep of a given LA and their number does not exceed the number of positive roots. Unfortunately, no explicit expression for the evaluation of generator matrix elements is available at present (see, however, [125]).

For the sake of simplicity we adhere to the usual abbreviation for the elementary lowering generators $E_{i+1, i}$, designating them by $f_{i}$, i.e., $f_{i} \equiv E_{i+1, i}$. The Verma monomials then have the following general form $[122,123]$ (referred to below as the V-version)

$$
\left(f_{1}^{a_{N}} f_{2}^{a_{N-1}} \cdots f_{n}^{a_{N-n+1}}\right)\left(f_{1}^{a_{N-n}} \cdots f_{n-1}^{a_{N-2 n+2}}\right) \cdots\left(f_{1}^{a_{3}} f_{2}^{a_{2}}\right) f_{1}^{a_{1}},
$$

where $N=n(n+1) / 2$. The parentheses in (5) are inserted in order to emphasize the structure of these monomials. The Verma inequalities for the irrep $\left[\lambda_{1}, \lambda_{2}, \cdots, \lambda_{n}\right] \equiv$ $\Lambda_{n}$ of the LA $A_{n} \equiv \mathfrak{s l}(n+1, \mathbb{C}),(n \geqslant 1)$ are given in Table 1 [123]. Note that the same basis set applies to the $\mathfrak{u}(n+1)$ or $\mathfrak{s u}(n+1)$ irrep $\left\langle m_{1}, m_{2}, \cdots, m_{n}, 0\right\rangle$ when we set $\lambda_{i}=m_{i}-m_{i+1}, i=1, \cdots, n$ (see the Appendix).

An alternative formulation due to Littelmann [125] uses a reversed order of gen- 
erators (referred to in the following text as the L-version), namely

$$
f_{1}^{a_{1}^{1}}\left(f_{2}^{a_{2}^{2}} f_{1}^{a_{1}^{2}}\right)\left(f_{3}^{a_{3}^{3}} f_{2}^{a_{2}^{3}} f_{1}^{a_{1}^{3}}\right) \cdots\left(f_{n}^{a_{n}^{n}} f_{n-1}^{a_{n-1}^{n}} \cdots f_{2}^{a_{2}^{n}} f_{1}^{a_{1}^{n}}\right)
$$

where the double-indexed exponents $a_{k}^{c}$ enable a simpler formulation of Verma inequalities, i.e.,

$$
0 \leqslant a_{k}^{c} \leqslant \min \left\{a_{k-1}^{c}+\lambda_{n-c+k}, a_{k+1}^{c+1}\right\}
$$

where

$$
a_{k}^{n+1}=+\infty, \quad a_{0}^{k}=0, \quad \text { and } \quad a_{k}^{c} \leqslant a_{k+1}^{c}, \forall k .
$$

The relationship of both alternative formulations has been outlined by Pošta and Havlíček (in particular see Lemma 3.5 of [126]). We shall see that both formulations lead to equivalent basis sets. We should also note that we could replace the elementary lowering generators by the raising ones acting on the lowest weight state.

\section{Simple applications}

\subsection{Four-electron singlet case}

As a simple example we first construct the Verma basis for the four-electron singlet $N=n=4$. The $\mathrm{SU}(2)$ two-column irrep of the spin part is $\left[2^{2}\right]$ and the corresponding conjugated orbital U(4) irrep is also $\left[2^{2}\right]$, i.e., it is characterized by the Young tableau with two boxes in the first two rows and no boxes in the third and the fourth row. The corresponding G-T U(4) irrep label $\left(m_{1} m_{2} m_{3} m_{4}\right)$ is (2200) and the related $A_{3} \equiv$ $\mathfrak{s l}(4, \mathbb{C})$ irrep label $\left\langle\lambda_{1} \lambda_{2} \lambda_{3}\right\rangle$ is $\langle 020\rangle$, with $\lambda_{i}=m_{i}-m_{i+1}$ (see the Appendix). The dimension of this irrep, according to Eq. (2) is 20. Since $n=4$, there are only three elementary lowering generators $f_{i} \equiv E_{i+1, i}$, namely $f_{1} \equiv E_{21}, f_{2} \equiv E_{32}$, and $f_{3} \equiv E_{43}$. Using the formulation of Li et al. [123] (version V), Eq. (5) and Table 1, the

relevant monomials have a general form $f_{1}^{a_{6}} f_{2}^{a_{5}} f_{3}^{a_{4}} f_{1}^{a_{3}} f_{2}^{a_{2}} f_{1}^{a_{1}}$, and the corresponding 
inequalities of Table 1 become

$$
\begin{aligned}
& 0 \leqslant a_{1} \leqslant \lambda_{1} \\
& 0 \leqslant a_{2} \leqslant \lambda_{2}+a_{1} \\
& 0 \leqslant a_{3} \leqslant \min \left\{\lambda_{2}, a_{2}\right\} \\
& 0 \leqslant a_{4} \leqslant \lambda_{3}+a_{2} \\
& 0 \leqslant a_{5} \leqslant \min \left\{\lambda_{3}+a_{3}, a_{4}\right\} \\
& 0 \leqslant a_{6} \leqslant \min \left\{\lambda_{3}, a_{5}\right\} .
\end{aligned}
$$

For our irrep [020] we have $\lambda_{1}=\lambda_{3}=0$ and $\lambda_{2}=2$, so that the Verma inequalities (25) become

$$
\begin{aligned}
& a_{1}=a_{6}=0 \\
& 0 \leqslant a_{2} \leqslant 2 \\
& 0 \leqslant a_{3} \leqslant \min \left\{2, a_{2}\right\}=a_{2} \\
& 0 \leqslant a_{4} \leqslant a_{2} \\
& 0 \leqslant a_{5} \leqslant \min \left\{a_{3}, a_{4}\right\},
\end{aligned}
$$

and Verma monomials take the form $f_{2}^{a_{5}} f_{3}^{a_{4}} f_{1}^{a_{3}} f_{2}^{a_{2}}$.

The list of possible nonzero exponents $a_{i}, i=2, \cdots, 5$ is shown in Fig. 1, indicating their systematic generation together with a lexical labeling of the resulting CSFs given in the bottom row. These exponents are then listed in the second column of Table 2. What we may call total or CSF weights $w$, given by the sum of the exponents $a_{i}$, $w=\sum_{k} a_{k}$, are given in the third column and the resulting monomials in the fourth column of the same table. To generate the basis vectors or CSFs of our irrep we let these monomials act on the HWS |HWS $\rangle$ of our (2200) irrep, namely the state that 
can be variously labeled as

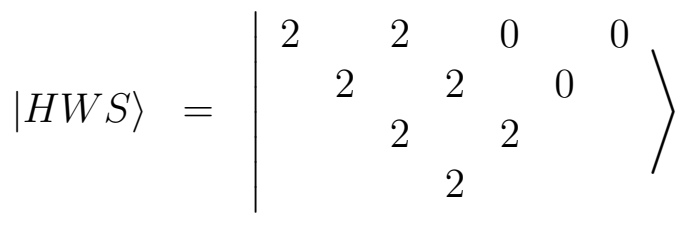

$$
\begin{aligned}
& \equiv\left[\begin{array}{lll}
2 & 0 & 2 \\
2 & 0 & 1 \\
2 & 0 & 0 \\
1 & 0 & 0
\end{array}\right] \equiv\left[\begin{array}{ll}
0 & 1 \\
0 & 1 \\
1 & 0 \\
1 & 0
\end{array}\right] \equiv\left[\begin{array}{ll}
0 & 0 \\
0 & 0 \\
1 & 1 \\
1 & 1
\end{array}\right] \equiv\left[\begin{array}{l}
0 \\
0 \\
2 \\
2
\end{array}\right] \\
& \equiv \begin{array}{|l|l|}
\hline 1 & 1 \\
\hline 2 & 2 \\
\hline
\end{array}=|1 \overline{1} 2 \overline{2}\rangle \text {. }
\end{aligned}
$$

Here in the first line there is the relevant G-T tableau, in the second row the ABC, $\Delta a \Delta c, \Delta a \overline{\Delta c}$ tableaux and the step number vector $\left[d_{i}\right]$, Eq. (3), and in the last row the Weyl tableau and $|1 \overline{1} 2 \overline{2}\rangle$ representing a CSF with doubly occupied first two orbitals. Note that this HWS is the same in all basis sets, specifically in both the G-T and Verma bases, which is not the case for some of the remaining basis vectors. The resulting (unnormalized) Verma basis CSFs $\left|v_{i}\right\rangle \equiv|i\rangle$ are then listed in the sixth column, while in the seventh column we indicate the ionicity $I$ of the CSFs interpreted as a VB wave functions. Another representation of the CSFs $|i\rangle$ in the form $|i\rangle=f_{j}|k\rangle$, implying their generic relationship, is listed in the fifth column.

The VB connection can be made even more apparent by considering a minimum basis set (MBS) PPP model of cyclobutadiene, in which case we can represent the basis states or CSFs $|i\rangle$ by VB structures shown in Fig. 2(a), where they are arranged according to their ionicity $I$, i.e., as the valence $(I=0)$, singly-ionic $(I=1)$, and doubly-ionic $(I=2)$ structures. Of course, in view of the point group symmetry of this model $\left(C_{4}\right.$ or $\left.D_{4 h}\right)$, the numbering of the atomic sites is irrelevant and the one employed here is indicated in Fig. 2(b). Clearly, a renumbering of the atomic sites will only permute the structures in the same row of Fig. 2(a).

In order to appreciate the structure of this basis set we display it in a diagrammatic form in Fig. 3 by arranging the CSFs by their total weights $w,\left(w=\sum_{k} a_{k}\right)$ as implied by their generic structure shown in the fifth column of Table 2. This graph is 
reminiscent of the so-called 'harmonic level excitation diagram' (HLED) of CAUGA [32] and we shall employ the same term here unless a confusion could arise. Note that the direction of the edges represents the relevant lowering generators $f_{i} \equiv E_{i, i+1},(i=$ 1,2 , and 3) as shown on the left hand side of the figure. The path connecting a given state $|i\rangle$ with the HWS $|1\rangle$ then implies the relevant generating Verma monomial, while the individual edges encode the $f_{j}$ 's given by the generic form $f_{j}|k\rangle$ of Table 2 . In fact, the HLED diagram also implies the relationship between various CSFs that can be connected via additional $f_{1}, f_{2}$, and $f_{3}$ edges. Thus, for example, we see that acting with $f_{1}$ on the 7 th CSF will yield the 9 th CSF, i.e., $f_{1}|7\rangle=|9\rangle$. Similarly, $f_{2}|7\rangle=|8\rangle, f_{2}|4\rangle=|5\rangle, f_{1}|8\rangle=|10\rangle$, etc., but $f_{2}|8\rangle \neq|15\rangle$. We do not indicate these additional relationships in Fig. 3 lest its essential basic structure be obscured.

Turning now to an alternative (reverse) formulation of Littelmann [125] and Pošta and Havlíček [126] (version L), Eqs. (6 - 8), the relevant monomials have the form $f_{1}^{a_{1}^{1}} f_{2}^{a_{2}^{2}} f_{1}^{a_{1}^{2}} f_{3}^{a_{3}^{3}} f_{2}^{a_{2}^{3}} f_{1}^{a_{1}^{3}}$ and the exponent determining inequalities, Eqs. (7) and (8), become

$$
\begin{aligned}
& 0 \leqslant a_{1}^{3} \leqslant \min \left\{a_{0}^{3}+\lambda_{1}, a_{2}^{4}\right\}=\min \{0, \infty\}=0 \\
& 0 \leqslant a_{2}^{3} \leqslant \min \left\{a_{1}^{3}+\lambda_{2}, a_{3}^{4}\right\}=\min \left\{a_{1}^{3}+2, \infty\right\}=a_{1}^{3}+2 \\
& 0 \leqslant a_{3}^{3} \leqslant \min \left\{a_{2}^{3}+\lambda_{3}, a_{4}^{4}\right\}=\min \left\{a_{2}^{3}, \infty\right\}=a_{2}^{3} \\
& 0 \leqslant a_{1}^{2} \leqslant \min \left\{a_{0}^{2}+\lambda_{2}, a_{2}^{3}\right\}=\min \left\{2, a_{2}^{3}\right\} \\
& 0 \leqslant a_{2}^{2} \leqslant \min \left\{a_{1}^{2}+\lambda_{3}, a_{3}^{3}\right\}=\min \left\{a_{1}^{2}, a_{3}^{3}\right\} \\
& 0 \leqslant a_{1}^{1} \leqslant \min \left\{a_{0}^{1}+\lambda_{3}, a_{2}^{2}\right\}=\min \left\{0, a_{2}^{2}\right\}=0,
\end{aligned}
$$

where on the rightmost side we have already used the fact that we consider the $A_{3}$ 
irrep $\Lambda_{3}=[020]$. Thus, finally, we have that

$$
\begin{aligned}
a_{1}^{1} & =a_{1}^{3}=0 \\
0 & \leqslant a_{2}^{3} \leqslant 2 \\
0 & \leqslant a_{3}^{3} \leqslant a_{2}^{2} \\
0 & \leqslant a_{1}^{2} \leqslant \min \left\{2, a_{2}^{3}\right\}=a_{2}^{3} \\
0 & \leqslant a_{2}^{2} \leqslant=\min \left\{a_{1}^{2}, a_{3}^{3}\right\}
\end{aligned}
$$

with the general monomial having the form $f_{2}^{a_{2}^{2}} f_{1}^{a_{1}^{2}} f_{3}^{a_{3}^{3}} f_{2}^{a_{2}^{3}}$.

We see that the inequalities (13) yield the exponents $a_{k}^{c}$ that have the same structure as those given by inequalities (10) for $a_{i}$ 's if we identify the exponents $a_{2}^{3}, a_{3}^{3}, a_{1}^{2}$, and $a_{2}^{2}$ with $a_{2}, a_{3}, a_{4}$, and $a_{5}$, respectively, so that the scheme of Fig. 1 applies here as well. The relevant monomials and the resulting CSFs are then listed in Table 3. Moreover, in view of the fact that $f_{1}$ and $f_{3}$ commute, since $\left[f_{1}, f_{3}\right]=\left[E_{21}, E_{43}\right]=0$, the monomials resulting from the reversed generator order (6) are the same as those resulting from the forward Verma scheme (5). Consequently, the use of the L-version (6) leads only to a reordering of the CSF states as implied by the index shown in the rightmost column of Tables 2 and 3 designated as $i^{\prime}$. Likewise, the HLED graph associated with the CSFs of Table 3 has the same structure as that in Fig. 3 with labels $i$ replaced by labels $i^{\prime}$ given in the last column of Table 3, so that the only effect is that the labels of Fig. 3 are symmetrically reflected about the vertical central axis, for which $i^{\prime}=i(i=1,2,3,9,10,15,14,16,19,20)$, while the remaining states are symmetrically reflected, i.e., $4 \leftrightarrow 7,5 \leftrightarrow 8,6 \leftrightarrow 12,11 \leftrightarrow 13$, and $17 \leftrightarrow 18$.

\subsection{Four-electron triplet case}

As another illustration let us consider the corresponding triplet case associated with the $\mathrm{U}(4)$ irrep $\langle 2110\rangle \equiv \Gamma(121)$ corresponding to the $A_{3}$ irrep $\Lambda_{3}=[101]$, so that $\lambda_{1}=\lambda_{3}=1$ and $\lambda_{2}=0$. Clearly, in this case we have $\operatorname{dim} \Gamma(121)=15$. The 
inequalities of Table 1 then yield

$$
\begin{aligned}
& 0 \leqslant a_{1} \leqslant 1 \\
& 0 \leqslant a_{2} \leqslant a_{1} \\
& 0 \leqslant a_{3} \leqslant \min \left\{0, a_{2}\right\}=0 \\
& 0 \leqslant a_{4} \leqslant 1+a_{2} \\
& 0 \leqslant a_{5} \leqslant \min \left\{1+a_{3}, a_{4}\right\} \\
& 0 \leqslant a_{6} \leqslant \min \left\{1, a_{5}\right\} .
\end{aligned}
$$

We thus see that in this case $a_{3}=0$, so that finally

$$
\begin{aligned}
& 0 \leqslant a_{1} \leqslant 1 \\
& 0 \leqslant a_{2} \leqslant a_{1} \\
& a_{3}=0 \\
& 0 \leqslant a_{4} \leqslant 1+a_{2} \\
& 0 \leqslant a_{5} \leqslant \min \left\{1, a_{4}\right\} \\
& 0 \leqslant a_{6} \leqslant \min \left\{1, a_{5}\right\}
\end{aligned}
$$

and the relevant Verma monomials have the form $f_{1}^{a_{6}} f_{2}^{a_{5}} f_{3}^{a_{4}} f_{2}^{a_{2}} f_{1}^{a_{1}}$.

Proceeding in the same way as in the singlet case we show the generation of possible values of the exponents $a_{i}$ in Fig. 4 and present them in a lexical order in the second column of Table 4 together with their total weights $w$. The recursive generation is indicated in the fifth column of Table 4 and the corresponding HLED diagram is shown in Fig. 5. The CSFs $|i\rangle$ are then listed in the last column of Table 4 and the pertinent cyclobutadiene VB structures are shown in Fig. 6.

\subsection{Ionic structures}

Consider, next, a singly ionized doublet state as described by the U(4) irrep $\langle 2100\rangle \equiv \Gamma(112)$ corresponding to the $A_{3}$ irrep $\Lambda_{3}=[110]$, so that $\lambda_{1}=\lambda_{2}=1$ and $\lambda_{3}=0$. Clearly, in this case we have $\operatorname{dim} \Gamma(112)=20$. Relying on the $\mathrm{V}$-version 
of Table 1, we find the following inequalities for the exponents $a_{i},(i=1, \cdots, 6)$

$$
\begin{aligned}
& 0 \leqslant a_{1} \leqslant 1 \\
& 0 \leqslant a_{2} \leqslant 1+a_{1} \\
& 0 \leqslant a_{3} \leqslant \min \left\{1, a_{2}\right\} \\
& 0 \leqslant a_{4} \leqslant a_{2} \\
& 0 \leqslant a_{5} \leqslant \min \left\{a_{3}, a_{4}\right\} \\
& 0 \leqslant a_{6} \leqslant 0,
\end{aligned}
$$

so that the pertinent Verma polynomials have the form $f_{2}^{a_{5}} f_{3}^{a_{4}} f_{1}^{a_{3}} f_{2}^{a_{2}} f_{1}^{a_{1}}$ since $a_{6}=0$. The nonvanishing exponents $a_{i}$ which generate the possible 20 states are found in the same way as in Figs. 1 and 4, and are listed in the second column of Table 5 together with the corresponding weights, Verma monomials, and the resulting CSF's. The fifth column labeled by $f_{j}|k\rangle$ implies the recursive buildup of the Verma basis as illustrated by the HLED diagram of Fig. 7(a).

Finally, let us consider a doubly-ionized singlet case or, correspondingly, a twoelectron ethylenic states as described by a four-orbital basis set, characterized by the $\mathrm{U}(4)$ irrep $\langle 2000\rangle \equiv \Gamma(103)$ corresponding to the $A_{3}$ irrep $\Lambda_{3}=[200]$, so that $\lambda_{1}=2$ and $\lambda_{2}=\lambda_{3}=0$. Clearly, in this case we have $\operatorname{dim} \Gamma(103)=10$. Relying again on the $\mathrm{V}$-version of Table 1 we find the following inequalities for the exponents $a_{i}$, $(i=1, \cdots, 6)$

$$
\begin{aligned}
& 0 \leqslant a_{1} \leqslant 2 \\
& 0 \leqslant a_{2} \leqslant a_{1} \\
& 0 \leqslant a_{3} \leqslant 0 \\
& 0 \leqslant a_{4} \leqslant a_{2} \\
& 0 \leqslant a_{5} \leqslant 0 \\
& 0 \leqslant a_{6} \leqslant 0,
\end{aligned}
$$

so that in this simplest case the Verma polynomials have the form $f_{3}^{a_{4}} f_{2}^{a_{2}} f_{1}^{a_{1}}$, since 
$a_{3}=a_{5}=a_{6}=0$. We thus easily find possible values for $a_{1}, a_{2}$ and $a_{4}$ listed in the second column of Table 6 , having the same structure as Tables 4 and 5 . The pertinent HLED is shown in Fig. 7(b). We note that in this case we would obtain the same result using the L-version in which case the exponents $a_{1}, a_{2}, a_{4}$ would be replaced by $a_{1}^{3}, a_{2}^{3}, a_{3}^{3}$, since in this case $a_{1}^{2}=a_{2}^{2}=a_{1}^{1}=0$, with pertinent polynomials having the form $f_{3}^{a_{3}^{3}} f_{2}^{a_{2}^{3}} f_{1}^{a_{1}^{3}}$.

\section{Discussion}

\subsection{Related basis sets}

As already pointed out above, the Verma bases for the $A_{n}$ irreps possess a number of desirable properties. Although their basis vectors are not necessarily mutually orthogonal, they are linearly independent and in the many-electron case are not tied up with any particular spin-coupling scheme, as is the case for the electronic G-T bases. Remarkably, however, they can be readily associated with the VB states which have a well known chemical interpretation, particularly for the $\pi$-electron systems with conjugated double bonds that play an important role in theoretical organic chemistry. Let us first, however, discuss other related basis sets, namely the so-called generator states of Matsen [9,114-116] and the CAUGA spinorial bases [32] that are also produced via a sequence of lowering generators applied to the HWS.

The generator states of Matsen [9,114-116] are produced by the action of weight lowering generators on the HWS of a given irrep. Matsen distinguishes the canonical generator states that are produced by a sequence of not-necessarily elementary lowering generators and the reduced generator states that are of the lowest degree in the generators (i.e., including nonelementary ones). His generator state basis is then represented by all reduced, canonical generator states. However, such a basis is not only nonorthogonal (for states of the same degree), but it is also overcomplete (i.e., linearly dependent, so that strictly speaking it is a spanning set rather than a basis). He thus invokes the Moshinsky-Nagel transformation $[48,49]$ to convert his generator 
basis to the orthonormal G-T basis. The whole procedure has been only illustrated on a couple of few-electron examples.

The CAUGA scheme $[32,83,101,117,118]$, on the other hand, exploits the imbedding of $\mathrm{U}(n)$ in a much larger group $\mathrm{U}\left(2^{n}\right)$ via the special orthogonal group $\mathrm{SO}(m)$, $m=2 n$ or $2 n+1$, and its covering group $\operatorname{Spin}(m)$. Again, one deals in reality with the corresponding LAs and exploits the imbedding of $\mathfrak{s o}(m)$ in the $2^{n}$-dimensional Clifford algebra $C_{n}$, relying on the CAUGA group chain (4). The $\mathrm{U}\left(2^{n}\right) \supset \mathrm{SO}(m)$ chain was first elucidated by Nikam et al. $[119,120]$. This approach works then with two-box spinorial states and the $\mathrm{SO}(m)$ - or, in fact $\mathrm{U}(n)$ - generators expressed in terms of the $\mathrm{U}\left(2^{n}\right)$ generators, which enables a trivial evaluation of the corresponding generator matrix elements. The CAUGA states may then be systematically generated by applying elementary lowering generators to the recursively generated states, starting with the HWS, and may be arranged into an appropriate CAUGA HLED diagram [32] (see also [127] for UGA HLED).

It is thus informative to compare the CAUGA HLED for the $\langle 2110\rangle$ irrep of U(4) in Fig. 6 of Ref. [32] with the present V-version in Fig. 5, as well as with UGA HLED in Fig. 5 of Ref. [32]. In particular, it should be noted that the states associated with the level $w=3$ in Fig. 5, that are associated with the nonorthogonal Kekulétype structures (cf. the first row in Fig. 6), correspond to the nonorthogonal twobox CAUGA states, in which case only the one associated with the leftmost path corresponds to a G-T state. The Schmidt orthogonalization of the remaining two states then yields the other G-T states as indicated in the HLED in Fig. 6 of [32]. In fact, this is the case in general: Clearly, the highest and the lowest weight states are the same as in the G-T case. Also, states associated with distinct levels of the HLED diagram (i.e., having a different weight $w$ ) are mutually orthogonal. However, those having the same weight are not necessarily orthogonal but, when suitably Schmidt orthogonalized, become equivalent to G-T states. See also the following Section and Fig. 9 for the benzene example. 


\subsection{Covalent states}

Let us recall that the VB approach is particularly useful for the description of planar $\pi$-electron systems with conjugated double bonds. Here the dominant role is played by non-ionic covalent structures, the most important ones being those of the Kekulé type followed by the Dewar-type structures. The same holds when using the VB-type formalism in CI calculations when one invariably has to truncate the FCI problem whose dimension rapidly increases with the size of the system. When handling ground states of closed-shell systems, the covalent structures play the dominant role. When a greater accuracy is required, one should also include ionic structures, in particular the mono-ionic (dipolar) ones. The states of higher ionicity than the dipolar ones are seldom required. For this very reason it would be useful to first identify the covalent states, followed eventually by the ionic ones. This selection can be best illustrated by considering simple $\pi$-electron model systems.

Consider, first, the singlet case of the $\langle 2200\rangle \mathrm{U}(4)$ irrep involving 20 CSFs listed in Tables 2 and 3. The corresponding VB structures - regarding the latter as the CSFs describing the $\pi$-electron model of cyclobutadiene - are shown in Fig. 2. Clearly, in this simple case only Kekulé-type covalent structures can arise. The HLED scheme of Fig. 3 then implies that the canonical Kekule structures, that are associated with the states $|10\rangle$ and $|15\rangle$, occur at the weight level $w=4$. In contrast, the G-T basis would involve only one of those structures, the other one being a non-canonical one shown in Fig. 8(c), representing a linear combination of canonical structures. Indeed, the linear dependence of the canonical and non-canonical covalent structures is symbolically represented by the relationship of Fig. 8 (up to the phase). Similarly, in the six-electron case, represented by the $\pi$-electron model of benzene (see below), all G-T covalent states except the one correspond to non-canonical structures as shown in Fig. 9 (cf. also Table III and Fig. 1 of Ref. [32]).

In view of the importance of covalent VB structures let us now turn our attention to finding a procedure of identifying these structures a priory, which can be useful 
when we wish to create a truncated basis set that is relevant from a chemical viewpoint. Of course, one can always write a simple code generating the entire Verma basis and then identify and select these structures. It is, however, instructive to see how we can find these states directly, at least in the case of simple $\pi$-electron model systems. We recall that the weight $w$ of a given state or CSF, which in turn defines the HLED level, is defined as the sum of the the exponents $a_{i}$ or $a_{k}^{c}$, i.e., $w=\sum_{i} a_{i}=\sum_{c, k} a_{k}^{c}$. This number also indicates the number of elementary lowering generators or elementary steps that are required to reach a given state starting from the HWS.

As an example consider a singlet ground state of a $\pi$-electron model of planar systems with conjugated double bonds having $n$ sites occupied by $N=n$ electrons or, correspondingly, ab initio models described by a minimum basis set (MBS). The relevant $\mathrm{U}(n)$ irreps are $\Gamma(a, b, c) \equiv\left\langle 2^{a} 1^{b} 0^{c}\right\rangle$, where $b=0, n=a+c$, and $N=$ $2 a$, so that $c=a$. The HWS $|1\rangle$ then involves doubly-occupied sites, i.e., $|1\rangle=$ $|1 \overline{1} 2 \overline{2} \cdots a \bar{a}\rangle$. The action of the elementary lowering generators $f_{i}=E_{i+1, i}$ may then be conveniently represented graphically, as illustrated in Fig. 10 for the three states of the cyclobutadiene model (Table 2) and, similarly, in Fig. 11 for the benzene covalent structures.

Since the covalent structures do not involve doubly-occupied sites, the required number of elementary lowering generators that are required to reach covalent states can be shown to be given by $w_{\text {cov }}=a^{2}, a=N / 2=n / 2$. This is most easily seen starting with the HWS of a cyclic polynomial $\mathrm{C}_{N} \mathrm{H}_{N}$ with the first $a$ doubly occupied sites and counting the number of required elementary translations along the $N$-gon to reach what will be called the highest covalent state (HCS) with singly occupied sites (as in the examples shown in Figs. 10 and 11). Counting then the number of required elementary steps or shifts we find that

$$
w_{\mathrm{cov}}=a+2 \sum_{i=1}^{a-1} i=a^{2} .
$$


In the case of ions, when we have more sites than electrons, this parameter provides only a lower bound for the number of elementary steps leading to covalent-type structures. Thus, the ionic covalent structures are no longer characterized by a single value of $w_{\mathrm{cov}}$.

To start with, let us describe how to generate what we have called above the HCS. As can be seen from Figs. 3, 5, 7 and 10, 11, we can reach covalent states via distinct routes starting with the HWS and applying appropriate strings of elementary lowering generators. One of these routes leads to the HCS $|i\rangle$ with the highest label $i$, and is generally given by the string

$a(a+1) \cdots(2 a-1) ;(a-1) a(a+1) \cdots(2 a-2) ;(a-2)(a-1) a \cdots(2 a-3) ; \cdots ; 12 \cdots(a-1) a$,

where each integer $i,(i=1,2, \cdots, n-1)$, represents a generator $f_{i}$. The semicolons then distinguish appropriate sequences of increasing integers [they are analogous to the parentheses in expressions (5) and (6)]. Thus, in the case of our four-electron example that is associated with the $\mathrm{U}(4)$ irrep $\langle 2200\rangle \equiv\left\langle 2^{2} 0^{2}\right\rangle \equiv \Gamma(202)$, we have $a=2$ and the string (19) becomes 2312, i.e., $f_{2} f_{3} f_{1} f_{2}$, corresponding to the state $|15\rangle$ characterized by the exponents $a_{i}=1, i=2,3,4,5$ (see Tables 2 and 3 and Figs. 1-3). In the case of the benzene model that is associated with the U(6) irrep $\left\langle 2^{3} 0^{3}\right\rangle \equiv \Gamma(303)$, we have $a=3$ and the corresponding string generating the HCS is $345 ; 234 ; 123$. In the general case, we can prove (19) by induction.

Now, since in the covalent states each site is occupied by a single electron, the only difference between the HCS and the other covalent states is the order of elementary generators constituting the generating string (19) for the HCS. We can thus generate the remaining covalent structures by moving the leftmost generator to the corresponding rightmost one thus raising its power. For example, in the cyclobutadiene case the HCS generating string 2312 becomes $312^{2}$, yielding the state $|10\rangle$ (cf. Fig. 9). Similarly, in the benzene case the HCS generating string 345234123 becomes $45234123^{2}$. Here, however, we can continue this process since in this case there are 
five covalent states, namely two Kekulé and three Dewar structures. Continuing the process we see that the rightmost generator to the left of the squared generator $3^{2}$ is 2 which, however, does not match the leftmost one which is 4 . In such a case we must generate two new states obtained by a translation of both generators, one by moving 2 resulting in the string $453412^{2} 3^{2}$ and another one by moving 4 and yielding $5234^{2} 123^{2}$. In the latter string there is no pair of identical generators to the left of the squared one, but in the first one we can again move 4 yielding $534^{2} 12^{2} 3^{2}$, thus obtaining all five covalent structures. The entire process is illustrated in Fig. 12. A larger example is displayed in Fig. 13.

In closing, we must note that is some cases the above outlined procedure may yield a seemingly overcomplete set of covalent states. This is not, however, the case because the superfluous string(s) will yield a vanishing result when acting on the HWS. As an example, consider the $\pi$-electron model of naphthalene (10 sites and 10 electrons, $N=n=10$, so that $a=5$ ), where we arrive to a covalent state associated with the string $8956784567^{2} 23456^{2} 123^{2} 4^{2} 5^{2}$. Following the above outlined procedure we can move 6 yielding $89578456^{2} 7^{2} 23456^{2} 123^{2} 4^{2} 5^{2}$. However, this string will yield a vanishing result when applied to the HWS. Pictorially one finds that after applying part of the above indicated string, namely $7^{2} 23456^{2} 123^{2} 4^{2} 5^{2}$, to the HWS, one obtains a structure with a singly occupied site 6 (using the standard site labeling this VB structure has doubly occupied sites 4, 5, and 8 and bonds 1-2 and 3-6), so that applying $6^{2} \equiv E_{7,6}^{2}$ to that structure gives a vanishing result.

\subsection{Extended basis sets}

So far we have considered classical VB structures with a single (orthogonal or nonorthogonal) AO per site involving the same number of electrons as sites or less in case of ions. In the MO context this would correspond to the use of a MBS, the smallest basis set possible. For a more accurate description one employs extended AO basis sets. In the MO context this generates a correspondingly larger set of virtual MOs 
(usually of the Hartree-Fock or Brueckner type). The reference state or the ground state CSF is then given by the HWS of a pertinent UGA irrep, and the remaining CSFs represent singly, doubly, etc. excited configurations relative to the HWS. There is no principal difficulty here except a rapid growth of the dimensionality of the FCI, requiring an inevitable truncation of the number of considered CSFs. One way to achieve this is to rely on the HLED scheme with a focus on its upper part.

In contrast, in VB approaches the most important CSFs are represented by covalent structures that appear in the middle of the Verma basis HLED. Consequently, a possible use of the extended basis sets in VB approaches is not straightforward and goes beyond the scope of this paper. Here, we will only point out one possible approach to this problem that is based on the shell-model partitioning of the AO basis set. For this purpose we exploit a linear span of an extended basis set having a subspace that is spanned by the lowest lying valence AOs on each site which is isomorphic with that spanned by the MBS. Such an extended basis set then involves additional AOs on each center and the corresponding VB approach will likely produce more accurate energies and wave functions, while still enabling a transparent, physically and chemically meaningful, interpretation. Of course, once we involve more than one AO per site we lose the correspondence between the singlet coupled AO pairs $\phi_{i}$, $\phi_{j}$ and the $i-j$ bonds, so that the bonds in what we referred to as the "covalent structures' may no longer represent covalent bonds in the standard chemical sense and the developments in Sect. 4.2 may not apply.

As a simple example, let us briefly consider one possible approach to the problem of extended basis sets using a triple-zeta (TZ) AO basis for a description of the ground state of the $\pi$-electron model of ethylene. We will thus employ the following shell-model labeling of a TZ basis, namely $\left\{\phi_{1}=2 p_{A}, \phi_{2}=2 p_{B}\right\},\left\{\phi_{3}=2 p^{\prime}{ }_{A}\right.$, $\left.\phi_{4}=2 p^{\prime}{ }_{B}\right\}$, and $\left\{\phi_{5}=2 p^{\prime \prime}{ }_{A}\right.$, and $\left.\phi_{6}=2 p^{\prime \prime}{ }_{B}\right\}$, representing the first, the second, and the third shells, respectively. The relevant unitary group is $\mathrm{U}(6)$ or $\mathrm{SU}(6)$ and the singlet ground state irrep is $\langle 2 \dot{0}\rangle \equiv[2]$ of dimension 21 (the symbol $\dot{0}$ indicates an 
appropriate number of zeroes which we drop for the sake of simplicity and indicate by using the square brackets). If desired, we could also think of a TZ model of the hydrogen molecule using the $1 s, 2 s$, and $3 s$ AO basis.

Clearly, it will be useful to consider subspaces spanned by CSFs that relate to the bonding of ethylenic orbitals which can be based on the following group chain

$$
\mathrm{U}(6) \supset \mathrm{U}(4) \otimes \mathrm{U}(2) \supset \mathrm{U}(2) \otimes \mathrm{U}(2) \otimes \mathrm{U}(2) .
$$

The overall $\mathrm{U}(6)$ irrep [2] may then be subduced with respect to the subgroups of this chain, obtaining

$$
[2] \downarrow U(4) \otimes U(2)=[2] \otimes[0]+[1] \otimes[1]+[0] \otimes[2]
$$

with corresponding dimensions

$$
21=10 \times 1+4 \times 2+1 \times 3
$$

since $\mathrm{U}(4)$ dimensions are $\operatorname{dim}[2]=10, \operatorname{dim}[1]=4$, and $\operatorname{dim}[0]=1$, while those of $\mathrm{U}(2)$ are $\operatorname{dim}[2]=3, \operatorname{dim}[1]=2$, and $\operatorname{dim}[0]=1$. Similarly, using the most relevant subgroup involving $\mathrm{U}(2)$ irreps gives

$$
\begin{aligned}
{[2] \downarrow \mathrm{U}(2) \otimes \mathrm{U}(2) \otimes \mathrm{U}(2)=} & ([2] \otimes[0]+[1] \otimes[1]+[0] \otimes[2]) \otimes[0] \\
& +([1] \otimes[0]+[0] \otimes[1]) \otimes[1] \\
& +[0] \otimes[0] \otimes[2],
\end{aligned}
$$

the corresponding dimensions being

$$
21=(3 \times 1+2 \times 2+1 \times 3) \times 1+(2 \times 1+1 \times 2) \times 2+1 \times 1 \times 3 .
$$

Let us point out here a formal similarity with the system partitioning within the UGA formalism $[65,81-86]$.

The defining inequalities for the exponents $a_{i}, i=1, \cdots, 15$, defining Verma mono- 
mials (V-version) $\left(f_{1}^{a_{15}} f_{2}^{a_{14}} f_{3}^{a_{13}} f_{4}^{a_{12}} f_{5}^{a_{11}}\right)\left(f_{1}^{a_{10}} f_{2}^{a_{9}} f_{3}^{a_{8}} f_{4}^{a_{7}}\right)\left(f_{1}^{a_{6}} f_{2}^{a_{5}} f_{3}^{a_{4}}\right)\left(f_{1}^{a_{3}} f_{2}^{a_{2}}\right) f_{1}^{a_{1}}$, are

$$
\begin{aligned}
& 0 \leqslant a_{1} \leqslant 2 \\
& 0 \leqslant a_{2} \leqslant a_{1} \\
& 0 \leqslant a_{4} \leqslant a_{2} \\
& 0 \leqslant a_{7} \leqslant a_{4} \\
& 0 \leqslant a_{11} \leqslant a_{7},
\end{aligned}
$$

with the remaining $a_{i}$ 's being equal to zero. Thus, the relevant Verma monomials have a simple form $f_{5}^{a_{11}} f_{4}^{a_{7}} f_{3}^{a_{4}} f_{2}^{a_{2}} f_{1}^{a_{1}}$. The list of possible nonvanishing exponents $a_{11} a_{7} a_{4} a_{2} a_{1}$ in a lexical order is given in the second column of Table 7 together with the corresponding weights $w_{i}$ in the third column. The fourth column then lists the Verma monomials and the generic build-up of the basis is indicated in the fifth column. The unnormalized basis vectors $\left|v_{i}\right\rangle$ (up to a phase) are then listed in the rightmost column.

The corresponding HLED (where we simultaneously display the relevant VB-like structures), arranged in a way to reflect the above given subduction (20), is shown in Fig. 14. The dashed rectangles, labeled by the capital letters A through D at the top left-hand-side corner, correspond to subspaces spanned by the enclosed states. The A, $\mathrm{B}$, and $\mathrm{C}$ blocks, each involving three structures, correspond to the irreps $[2] \otimes[0] \otimes[0]$, $[0] \otimes[2] \otimes[0]$, and $[0] \otimes[0] \otimes[2]$ of dimension 3, while those involving four structures, labeled by D, E, and F, correspond to irreps irreps $[1] \otimes[1] \otimes[0],[1] \otimes[0] \otimes[1]$, and $[0] \otimes[1] \otimes[1]$, respectively. This also implies that in the $\mathrm{U}(4) \otimes \mathrm{U}(2)$ subduction (21), the irrep [2] $\otimes[0]$ is associated with the 10 states constituting the blocks A, B, and $\mathrm{D}$, the irrep $[1] \otimes[1]$ with the blocks $\mathrm{E}$ and F, and the irrep $[0] \otimes[2]$ with the block C. Note that the block A represents the FCI within the MBS involving $\phi_{1}$ and $\phi_{2}$ with one covalent structure of the Heitler-London type and the two doubly-ionic structures. Similarly, B and C blocks are associated with the second and the third shells, respectively. The blocks D, E, and F then represent the inter-shell interactions. 
Clearly, the block F associated with the interaction between the second and the third shell will be of a secondary importance, as will likely be also the block E and the ionic structures in the second and third shell (blocks B and C). Note also that we can identify the HWS for each block. Thus, e.g., the HWS that is associated with the irrep $[2] \otimes[0] \otimes[0]$ is associated with the rightmost structure in the block A of Fig. 14 involving $\phi_{1}$ and $\phi_{2}$, i.e., the state $|1 \overline{1}\rangle$. In this way one can identify the role of individual VB structures and exploit this information for a physically and chemically meaningful truncation of the CI problem..

\section{Conclusions}

Thanks to the pioneering work of Heitler and London [128] and subsequent developments due to Pauling's school [129], the VB theory stood at the cradle of quantum chemistry. It rationalized for the first time the nature of chemical bonding on the basis of quantum mechanics and its qualitative version in the form of the so-called resonance theory, as advanced by Wheland $[130,131]$ in the West and Syrkin and Djatkina [132] in the East, was enthusiastically embraced especially by organic chemists, thanks to its intuitive appeal enabling a description of the molecular structure and reaction pathways. Nonetheless, it was later eclipsed by the MO theory and formalism due to a number of its difficulties, such as the neglect of overlap integrals, the 'nightmare' of the inner shells and, especially, the so-called N! catastrophe. Yet, it has not lost its appeal and potential usefulness. We thus find it quite remarkable that the VB structures naturally arise when one employs the so-called Verma bases for the $\mathrm{U}(n)$ or a corresponding $\mathfrak{s l}(n-1, \mathbb{C})$ irreps when relying on the UGA formalism.

Of course, the current exploitations of UGA always rely on the MO formalism, be it within the $\mathrm{CI}$ or the $\mathrm{CC}$ context, and exploit a formalism that is based on the G-T subgroup chain (1). The resulting spin-free CSFs are then related with the Yamanouchi-Kotani coupling scheme. In contrast, the Verma bases - which are of a much recent date than the G-T ones - are independent of any a priori coupling scheme 
or subgroup chain. As pointed out in Sect. 2, the Verma bases possess a number of desirable properties, being eigenvectors of the Cartan subalgebra and thus independent of any fixed subalgebra or subgroup chain, thus being free to adaptation with respect to physically relevant subgroups as, for example, demonstrated in Sect. 4.3. The main drawback of Verma bases is the fact that, so far, no explicit formulas are available for the evaluation of matrix elements of $\mathrm{U}(n)$ generators. Although this difficulty can be overcome in various ways, such as by relying on a connection with the permutation group $S_{n}$ or even with the G-T states via orthogonalization, this is definitely a disadvantage when compared with the efficient algorithms developed in UGA for the relevant two-column $\mathrm{U}(n)$ irreps via the segmentation or within the CAUGA, where the matrix element evaluation is trivial. Yet the Verma bases could be beneficial thanks to their relationship with the VB structures by generating the relevant covalent states in various VB schemes. It would also be of interest to explore their deeper relationship with the G-T and CAUGA states.

\section{Appendix}

We briefly recall a few basic facts concerning the structure of Lie algebras (LAs) and point out some relevant concepts concerning their representations. In general, an $n$-dimensional LA $\mathfrak{g}$ is an $n$-dimensional vector space equipped with a Lie product $[\cdot, \cdot]: \mathfrak{g} \times \mathfrak{g} \rightarrow \mathfrak{g}$, which is anti-commutative and satisfies the Jacobi identity. A general linear algebra $\mathfrak{g} \equiv \mathfrak{g l}(\mathcal{V})$ of an $n$-dimensional vector space $\mathcal{V}$ over $\mathbb{R}$ or $\mathbb{C}$ is then an algebra of endomorphisms of $\mathcal{V}$, End $\mathcal{V}=\{X \mid X: \mathcal{V} \rightarrow \mathcal{V}, X$ linear $\}$, $\operatorname{dim}($ End $\mathcal{V})=n^{2}$, equipped with a Lie product or a bracket $[X, Y]=X Y-Y X$. The matrix form of $\mathfrak{g l}(\mathcal{V})$ results when we fix a basis for $\mathcal{V}$, in which case we can identify $\mathfrak{g l}(\mathcal{V})$ with a set of $n \times n$ real or complex matrices, and we denote this LA as $\mathfrak{g l}(n, \mathbb{R})$ or $\mathfrak{g l}(n, \mathbb{C})$, respectively. A standard basis is then given by matric units $E_{i j}$, often referred to as generators,

$$
E_{i j}=\left\|e_{k l}\right\|, \quad e_{k l}=\delta_{k i} \delta_{l j} .
$$


Since $E_{i j} E_{k l}=\delta_{j k} E_{i l}$, the Lie product for generators becomes

$$
\left[E_{i j}, E_{k l}\right]=\delta_{j k} E_{i l}-\delta_{l i} E_{k j}
$$

Here $\delta_{i j}$ designates the Kronecker delta. Introducing a standard basis $\left\{e_{i}\right\}$ in $\mathcal{V}$, $e_{i}=(\cdots, 1, \cdots)^{t}$ with one in the $i$-th position and zeroes elsewhere, the superscript $t$ indicating a transposition, we have that $E_{i j} e_{k}=\delta_{j k} e_{i}$.

A non-abelian LA $([\mathfrak{g}, \mathfrak{g}] \neq 0)$ is called simple if it does not contain any nontrivial ideals and all complex semi-simple LAs are direct sums of simple LAs. There are four infinite families of semi-simple LAs $\left(A_{n}, B_{n}, C_{n}\right.$, and $\left.D_{n}\right)$ and five exceptional ones. We are particularly interested in special linear LAs $\mathfrak{s l}(n, \mathbb{C})$ of traceless $n \times n$ matrices over $\mathbb{C}$, and related unitary and special unitary LAs $\mathfrak{u}(n)$ and $\mathfrak{s u}(n)$ of skew-Hermitian and traceless skew-Hermitian matrices, respectively, namely

$$
\begin{aligned}
\mathfrak{s l}(n, \mathbb{C}) & =\{X \in \mathfrak{g l}(n, \mathbb{C}) \mid \operatorname{Tr} X=0\}, \\
\mathfrak{u}(n) & =\left\{X \in \mathfrak{g l}(n, \mathbb{C}) \mid X+X^{\dagger}=0\right\}, \\
\mathfrak{s u}(n) & =\left\{X \in \mathfrak{g l}(n, \mathbb{C}) \mid X+X^{\dagger}=0 \text { and } \operatorname{Tr} X=0\right\},
\end{aligned}
$$

the dagger indicating a Hermitian conjugate. Note that $\mathfrak{u}(n)$ and $\mathfrak{s u}(n)$ are real LAs and $\mathfrak{s l}(n, \mathbb{C})$ represents a complexification of $\mathfrak{s u}(n)$, i.e., $\mathfrak{s l}(n, \mathbb{C})=[\mathfrak{s u}(n)]^{\mathbb{C}}$. Consequently, there is a close relationship between their representations.

Of special interest for us are the $A_{n}$ or $\mathfrak{s l}(n, \mathbb{C})$ LAs, i.e.,

$$
A_{n} \equiv \mathfrak{s l}(n+1, \mathbb{C}), \quad \operatorname{dim} A_{n}=n(n+2),
$$

with a standard basis given by $n(n+1)$ off-diagonal $E_{i j},(1 \leq i, j \leq(n+1), i \neq j)$ and $n$ diagonal $H_{i}=E_{i i}-E_{i+1, i+1},(1 \leq i \leq n)$ generators. The latter span the corresponding Cartan subalgebra $\mathfrak{h}$ of all diagonal matrices $H$ in $\mathfrak{g} \equiv \mathfrak{s l}(n, \mathbb{C})$, i.e., $H \in \mathfrak{h}, H=\left\|h_{i} \delta_{i j}\right\|=\sum_{j=1}^{n} h_{j} E_{j j}, \operatorname{Tr}(H)=\sum_{i=1}^{n} h_{i}=0$. The dimension of the Cartan subalgebra $\operatorname{dim} \mathfrak{h}$ is called the rank of the LA. Clearly, $\mathfrak{h}$ is abelian and thus nilpotent, and its dual $\mathfrak{h}^{*}$ is given by a span of linear functionals $L_{i}$ on $\mathfrak{h}$ such that

$$
L_{i}(H)=L_{i} \sum_{j=1}^{n} h_{j} E_{j j}=h_{i}, \quad H \in \mathfrak{h} .
$$


The trace condition implies that $\sum_{i=1}^{n} L_{i}=0$. Note that the individual $E_{i i} \notin \mathfrak{h}$ in view of the trace condition.

Recall that a representation (rep) $\pi$ of a LA $\mathfrak{g}$ on a vector space $\mathcal{V}$ is a LA homomorphism $\pi: \mathfrak{g} \rightarrow \mathfrak{g l}(\mathcal{V})$, i.e., a linear transformation of LAs preserving the Lie product $\pi([A, B])=[\pi(A), \pi(B)]=\pi(A) \pi(B)-\pi(B) \pi(A)$. As above, $\mathfrak{g l}(\mathcal{V})$ denotes the space of endomorphisms of $\mathcal{V}$, End $\mathcal{V}$, i.e., the space of all linear maps $\phi: \mathcal{V} \rightarrow \mathcal{V}$, with a Lie product defined by a commutator $[X, Y]:=X Y-Y X$. By abuse of terminology, one often refers to $\mathcal{V}$ itself as a rep. More appropriately, however, the vector space $\mathcal{V}$ together with the rep $\pi$ constitute a $\mathfrak{g}$-module, i.e., a vector space together with a bilinear map $\mathfrak{g} \times \mathcal{V} \rightarrow \mathcal{V}$ defined by $[X, Y] \cdot v=X(Y \cdot v)-Y(X \cdot v)$.

Thus, $\mathfrak{g} \equiv \mathfrak{g l}(n, \mathbb{C})$ may be regarded as a representation of $\mathfrak{g l}(\mathcal{V})$ on a vector space $\mathcal{V}$ of $n \times n$ matrices or, equivalently, as a $\mathfrak{g}$-module. It is usually referred to as a standard representation. Let us note here that in physics one often does not distinguish between groups and corresponding algebras and talks about a representation of a group (e.g., as in the unitary group approach [1-22]) while relying on the corresponding LA.

Another useful concept is that of the adjoint representation ad,

$$
\operatorname{ad}: \mathfrak{g} \rightarrow \operatorname{Der} \mathfrak{g}: A \mapsto \operatorname{ad} A
$$

where ad $A$ acts as

$$
\operatorname{ad} A(B)=[A, B]
$$

Derivation of $\mathfrak{g}$, Der $\mathfrak{g}$, is a set of linear maps $\phi$ satisfying the usual product rule $\phi(A B)=A \phi(B)+\phi(A) B$. Clearly, ad is a derivation in view of the Jacobi identity, is linear, and preserves the bracket, i.e., $[\operatorname{ad} A, \operatorname{ad} B](C)=\operatorname{ad}[A, B](C)$.

Now, for semi-simple LAs all finite-dimensional reps are reducible. The constituting irreducible reps (irreps) may then be classified by their weights $\lambda$, representing the elements of $\mathfrak{h}^{*}$ resulting from a diagonalization of the $\mathfrak{h}$-action

$$
H v=\lambda(H) v, \quad v \in \mathcal{V}_{\lambda} \subset \mathcal{V}, H \in \mathfrak{h}
$$


Here we recall that any set of commuting operators or linear transformations on $\mathcal{V}$ can be simultaneously diagonalized. The spaces $\mathcal{V}_{\lambda}$,

$$
\mathcal{V}_{\lambda}:=\{v \in \mathcal{V} \mid H \cdot v=\lambda(H) v, \forall H \in \mathfrak{h}\}
$$

are called weight spaces and their non-zero elements are referred to as weight vectors. The linear functional $\lambda(H)$ on $\mathfrak{h}$ associated with a non-zero weight space is then called a weight of the rep $\mathcal{V}$. Thus, if $\left\{H_{1}, H_{2}, \cdots, H_{n}\right\}$ is a basis for $\mathfrak{h}$, a weight of a rep $(\pi, \mathcal{V})$ of $\mathfrak{g}$ is a set of simultaneous eigenvalues $\left\{\lambda_{1}, \lambda_{2}, \cdots, \lambda_{n}\right\}$ for commuting operators $\pi\left(H_{1}\right), \pi\left(H_{2}\right), \cdots, \pi\left(H_{n}\right)$.

Any rep $\mathcal{V}$ may then be expressed as a direct sum of weight spaces $\mathcal{V}=\bigoplus_{\lambda \in h^{*}} \mathcal{V}_{\lambda}$ Moreover, one can introduce a partial order on the set of weights that is based on such a partial ordering (see, e.g., [133]). With the highest weight $\lambda$ is then associated the highest weight space $\mathcal{V}_{\lambda}$ spanned by vectors $v \in \mathcal{V}$ that are annihilated by raising generators $E_{i j}, i<j$. By considering the real form $\mathfrak{h}_{0}$ of $\mathfrak{h}$, one can show that every weight on $\mathfrak{h}_{0}$ is real and, in fact, algebraically integral. The highest weights then uniquely label finite-dimensional irreps.

The weights of the adjoint rep are called roots. We find that the generators $E_{i j}$ are eigenvectors for the adjoint action of $H \in \mathfrak{h}$, i.e.,

$$
\operatorname{ad}(H) E_{i j}=\left[H, E_{i j}\right]=\left(h_{i}-h_{j}\right) E_{i j}=\left(L_{i}-L_{j}\right)(H) E_{i j}
$$

yielding the roots

$$
\lambda_{i j}=L_{i}-L_{j}, \quad i \neq j
$$

and the one-dimensional root spaces $\mathfrak{g}_{\lambda_{i j}}=\mathbb{C} E_{i j}$. The set of roots $R$ spans a lattice called the root lattice $\Lambda$. We distinguish positive roots $\lambda_{i j} \in R^{+}$when $i<j$, i.e., $R^{+}=\left\{\lambda_{i j}=L_{i}-L_{j} \mid i<j\right\}$ and negative roots belonging to $R^{-}=\left\{\lambda_{i j} \mid i>\right.$ $j$ \}. The former ones are associated with raising generators $E_{i j},(i<j)$, spanning the nilpotent subalgebra of upper triangular matrices $\mathfrak{g}^{+}$and the latter ones with lowering generators $(i>j)$ spanning the subalgebra of lower-triangular matrices 
$\mathfrak{g}^{-}$. This yields a Cartan decomposition

$$
\mathfrak{g}=\mathfrak{h} \bigoplus_{\lambda \in R \subseteq \mathfrak{h}^{*}} \mathfrak{g}_{\lambda}=\mathfrak{h} \oplus \mathfrak{g}^{+} \oplus \mathfrak{g}^{-}
$$

where, in general,

$$
\mathfrak{g}_{\lambda}=\{X \in \mathfrak{g} \mid \operatorname{ad} H(X)=\lambda(H) X ; \forall H \in \mathfrak{h}\},
$$

and, for semi-simple algebras, $\operatorname{dim} \mathfrak{g}_{\lambda}=1$. Generators involving neighboring indices, i.e., $E_{i, i+1}$ and $E_{i+1, i}$, are referred to as the elementary raising and lowering generators, respectively.

Now, if $X \in \mathfrak{g}_{\lambda}$, then $\operatorname{ad}(X)$ maps $\mathfrak{g}_{\mu}$ into $\mathfrak{g}_{\lambda+\mu}$ since

$$
\begin{aligned}
\operatorname{ad} H \operatorname{ad} X(Y) & =\operatorname{ad} X \operatorname{ad} H(Y)+\operatorname{ad}[H, X](Y) \\
& =[\mu(H)+\lambda(H)] \operatorname{ad} X(Y), \quad X \in \mathfrak{g}_{\lambda}, Y \in \mathfrak{g}_{\mu} .
\end{aligned}
$$

Note that if $\mu=-\lambda$, then $\left[\mathfrak{g}_{\lambda}, \mathfrak{g}_{-\lambda}\right] \subset \mathfrak{h}$ and we can identify a subalgebra

$$
\mathfrak{s}_{\lambda}=\mathfrak{g}_{\lambda} \oplus \mathfrak{g}_{-\lambda} \oplus\left[\mathfrak{g}_{\lambda}, \mathfrak{g}_{-\lambda}\right],
$$

which is isomorphic with $\mathfrak{s l}(2, \mathbb{C})$. Thus, $\mathfrak{g}$ is spanned by imbedded copies of $\mathfrak{s l}(2, \mathbb{C})$ LAs.

Specifically for $\mathfrak{s l}(n, \mathbb{C})$ the eigenvectors of $\operatorname{ad}(\mathfrak{h})$ are the generators $E_{i j}$ with eigenvalues $\left(L_{i}-L_{j}\right)$ as implied by Eq. (35) and we have a decomposition

$$
\mathfrak{s l}(n, \mathbb{C})=\mathfrak{h} \bigoplus_{i \neq j} \mathbb{C} E_{i j}
$$

Similarly to Eq. (40) we now have that $\mathfrak{s}_{L_{i}-L_{j}}=\mathbb{C}\left\langle E_{i j}, E_{j i}, H_{i}-H_{j}\right\rangle \cong \mathfrak{s l}(2, \mathbb{C})$ for distinct $\left(H_{i}-H_{j}\right)$. The weight lattice is $\mathbb{Z}\left\langle L_{1}, L_{2}, \cdots, L_{n}\right\rangle$ and the root lattice is the sublattice spanned by $\lambda_{i j}=\left(L_{i}-L_{j}\right)$. As pointed out above one can introduce an ordering of positive roots $R^{+}$and in view of the fact that $\mathfrak{g}$ is spanned by $\mathfrak{s l}(2, \mathbb{C})$ subalgebras it is intuitively not surprising that these weights are integral. Roots associated with elementary generators $E_{i, i \pm 1}, i=1,2, \cdots, n-1$ are then referred to 
as simple roots. In general, a simple root is a positive root that cannot be written as a sum of positive roots; e.g., $\lambda_{13}$ of $\mathfrak{s l}(3, \mathbb{C})$ is not simple, since $\lambda_{13}=\lambda_{12}+\lambda_{23}$. Since $\sum_{i=1}^{n} L_{i}=0$, one also introduces the so-called fundamental dominant weights

$$
\omega_{i}=\sum_{j=1}^{i} L_{j}, \quad i=1,2, \cdots, n-1,
$$

representing the edges of the Weyl chamber $W$. Note that since $\sum_{i=1}^{n} L_{i}=0$, we have that $\omega_{n-1}=-L_{n}$.

We thus see that general semi-simple LAs consist of $\mathfrak{s l}(2, \mathbb{C})$ subalgebras. We recall here that for $A_{1} \equiv \mathfrak{s l}(2, \mathbb{C})$ we can choose a basis

$$
x \equiv E_{12}=\left(\begin{array}{ll}
0 & 1 \\
0 & 0
\end{array}\right), \quad y \equiv E_{21}=\left(\begin{array}{ll}
0 & 0 \\
1 & 0
\end{array}\right), \quad \text { and } \quad h \equiv E_{11}-E_{22}=\left(\begin{array}{cc}
1 & 0 \\
0 & -1
\end{array}\right)
$$

yielding the commutation relations

$$
[h, x]=2 x,[h, y]=-2 y, \text { and }[x, y]=h .
$$

The linear functional $\lambda$ on $\mathfrak{h}=\{h\}$ is in this case completely determined by its value $\lambda(h)=\lambda$ at the basis vector $h$.

Now, for $v \in \mathcal{V}_{\lambda}$ we find that $x \cdot v \in \mathcal{V}_{\lambda+2}$ and $y \cdot v \in \mathcal{V}_{\lambda-2}$ since

$$
h \cdot(x \cdot v)=[h, x] \cdot v+x \cdot h \cdot v=2 x \cdot+\lambda x \cdot v=(\lambda+2) x \cdot v,
$$

and similarly for $y \cdot v$. It may be shown that $\lambda$ is integral and with a suitable normalization we find that for an irreducible module $\mathcal{V}$ for $\mathfrak{g}=\mathfrak{s l}(2, \mathbb{C})$ we have that $\mathcal{V}=\bigoplus_{\lambda} \mathcal{V}_{\lambda}, \lambda=m,(m-2), \cdots,-(m-2),-m$, with $\operatorname{dim} \mathcal{V}_{\lambda}=1$ for all $\lambda$ such that $\mathcal{V}_{\lambda} \neq 0$, so that $\operatorname{dim}(\mathcal{V})=m+1$. One can work out explicit formulas for the action of $x, y$ and $h$ once we choose a suitable basis for $\mathcal{V}$, as is well known from the second quantization approach to a harmonic oscillator. An equivalent development can be made for $\mathfrak{s u}(2)$ using Pauli matrices as a basis.

Recall that when dealing with the $\mathfrak{u}(n)$ or $\mathfrak{g l}(n, \mathbb{C})$ irreps, the relevant Cartan subalgebra $\mathfrak{h}$ is spanned by the diagonal $E_{i i}$ generators, $\mathfrak{h}=\left\{E_{11}, E_{22}, \cdots, E_{n n}\right\}$, 
often referred to in this context as weight generators. A given irrep $\pi(\lambda)$ then contains a unique (up to a scalar multiple) vector $\xi \neq 0$, referred to as the highest weight vector or state, such that $E_{i i} \xi=\lambda_{i} \xi, i=1, \cdots, n$ and $E_{i j} \xi=0$ for all $1 \leqslant i<j \leqslant n$. The $\mathfrak{u}(n)$ irreps are then in a one-to-one correspondence with $n$-tuples $\lambda=\left(\lambda_{1}, \lambda_{2}, \cdots, \lambda_{n}\right)$ such that $\lambda_{i}-\lambda_{i+1} \in \mathbb{Z}^{+}, i=1, \cdots, n-1$, and $\mathbb{Z}^{+}=\{i \in \mathbb{Z} \mid i \geqslant 0\}$, while in general $\lambda_{i} \in \mathbb{C}$. Note that for the purposes of G-T basis labeling the highest weight $\lambda$ 's are usually designated by $\mathbf{m}_{n}$, so that $\pi(\lambda) \equiv \Gamma\left(\mathbf{m}_{n}\right)$ and $\lambda=\left(\lambda_{1}, \lambda_{2}, \cdots, \lambda_{n}\right) \equiv\left(m_{1 n}, m_{2 n}, \cdots, m_{n n}\right) \equiv \mathbf{m}_{n}$. Now considering an automorphism $E_{i j} \rightarrow E_{i j}+a \delta_{i j}, a \in \mathbb{C}$ which preserves the $\mathfrak{u}(n)$ or $\mathfrak{g l}(n, \mathbb{C})$ commutation relations (i.e., the structure constants), we see that the irreps $\Gamma\left(\mathbf{m}_{n}\right)$ and $\Gamma\left(\mathbf{m}_{n}^{\prime}\right)$, labeled by the highest weights $\mathbf{m}_{n}=\left(m_{1 n}, m_{2 n}, \cdots, m_{n n}\right)$ and $\mathbf{m}_{n}^{\prime}=\left(m_{1 n}^{\prime}, m_{2 n}^{\prime}, \cdots, m_{n n}^{\prime}\right)$, where $m_{i n}^{\prime}=m_{i n}+a$, are simply related (see, e.g., p. 154 and Appendix A of [3]), while for the corresponding irreps of $\mathfrak{s l}(n, \mathbb{C})$ or $\mathfrak{s u}(n)$ are equivalent, i.e., $\Gamma\left\{\mathbf{m}_{n}\right\} \cong \Gamma\left\{\mathbf{m}_{n}^{\prime}\right\}$ (in fact, they are identical). Thus, without restricting generality, we can always choose $a=-m_{n n}$, so that the last component of the weight vector $\mathbf{m}_{n}$ will vanish, providing a unique labeling for these irreps, i.e., $\mathbf{m}_{n}=\left(m_{1 n}-m_{n n}, m_{2 n}-m_{n n}, \cdots, m_{n-1, n}-m_{n n}, 0\right)$.

Now the unique rep of $\mathfrak{s l}(n, \mathbb{C})$ with weight

$$
\sum_{i=1}^{n} b_{i} \lambda_{i}, \quad b_{i} \geq b_{i+1}, \quad i=1,2, \cdots, n-1,
$$

may be rewritten in terms of fundamental weights $\left\{\omega_{i}\right\}$, Eq. (42), as $\sum_{i=1}^{n-1} a_{i} \omega_{i}$ and designated as $\Lambda_{n-1} \equiv\left[a_{1}, a_{2}, \cdots, a_{n-1}\right]$. Comparing the latter form with that of Eq. (46), we require that $\sum_{j=i}^{n-1} a_{j}=b_{i}-b_{n},(i=1,2, \cdots, n-1)$, yielding the rep $\Lambda_{n-1} \equiv\left[a_{1}, a_{2}, \cdots, a_{n-1}\right]$ with $a_{i}=b_{i}-b_{i+1},(i=1,2, \cdots, n-1)$. In this notation, the $\mathfrak{u}(n)$ irrep $\left\langle\mu_{1}, \mu_{2}, \cdots, \mu_{n-1}, \mu_{n}\right\rangle$ can be considered as the $\mathfrak{s l}(n, \mathbb{C})$ irrep $\left[\mu_{1}-\mu_{2}, \mu_{2}-\mu_{3}, \cdots, \mu_{n-1}-\mu_{n}\right]$, resulting in a standard notation for the $A_{n}$ LAs.

Acknowledgments Past support by NSERC (J.P.) is gratefully acknowledged. Authors 
are also indebted to Professor Jiří Patera of the Centre de Recherches Mathématiques of the Université de Montréal for drawing their attention to Verma's work and to Dr. Jason Stuber for his kind help with the preparation of the manuscript.

\section{References}

[1] J. Paldus, J. Chem. Phys. 61, 5321 (1974)

[2] J. Paldus, Phys. Rev. A 14, 1620 (1976)

[3] J. Paldus, in Theoretical Chemistry: Advances and Perspectives, vol. 2, ed. by H. Eyring, D. J. Henderson (Academic, New York, 1976) pp. 131-290

[4] I. Shavitt, Int. J. Quantum. Chem. Symp. 11, 131 (1977)

[5] I. Shavitt, Int. J. Quantum. Chem. Symp. 12, 5 (1978)

[6] J. Paldus, in Lecture Notes in Chemistry, no. 22, ed. by J. Hinze (Springer, Berlin, 1981) pp. 1-50

[7] I. Shavitt, in Lecture Notes in Chemistry, no. 22, ed. by J. Hinze (Springer, Berlin, 1981) pp. 51-99

[8] M. A. Robb, U. Niazi, Comp. Phys. Rep. 1, 127 (1984)

[9] F. A. Matsen, R. Pauncz, The Unitary Group in Quantum Chemistry (Elsevier, Amsterdam, 1986)

[10] J. Paldus, in Mathematical Frontiers in Computational Chemical Physics, IMA Series, vol. 15, ed. by D. G. Truhlar (Springer, Berlin, 1988) pp. 262-299

[11] I. Shavitt, in Mathematical Frontiers in Computational Chemical Physics, IMA Series, vol. 15, ed. by D. G. Truhlar (Springer, Berlin, 1988) pp. 300-349

[12] M. A. Robb, Theor. Chem. Acc. 103, 317 (2000) 
[13] J. Paldus, in Handbook of Atomic, Molecular, and Optical Physics, chap. 4, 2nd ed., ed. by G. W. F. Drake (Springer, Berlin, 2006) pp. 101-114

[14] H. Baker, M. A. Robb, Mol. Phys. 50, 1077 (1983)

[15] J. Paldus, B. Jeziorski, Theeor. Chim. Acta 73, 81 (1988)

[16] X. Li, J. Paldus, J. Chem. Phys. 101, 8812 (1994)

[17] X. Li, J. Paldus, Chem. Phys. Lett. 231, 1 (1994)

[18] B. Jeziorski, J. Paldus, P. Jankowski, Int. J. Quantum. Chem. 56, 129 (1995)

[19] J. Paldus, X. Li, Can. J. Chem. 74, 918 (1996)

[20] P. Jankowski, B. Jeziorski, J. Chem. Phys. 111, 1857 (1999)

[21] J. Paldus, X. Li, Adv. Chem. Phys. 110, 1 (1999); sec. II.D.3.2 and loc. cit.

[22] X. Li, J. Paldus, J. Mol. Struct. (тheоchem) 527, 165 (2000)

[23] R. McWeeny, Methods in Molecular Quantum Mechanics, 2nd ed., chap. 10 (Academic, New York, 1989)

[24] F. W. Bobrowicz, W. A. Goddard III, in Methods of Electronic Structure Theory, ed. by H. F. Schaefer III (Plenum, New York, 1977) pp. 79-127

[25] D. L. Cooper, J. Gerratt, M. Raimondi, Adv. Chem. Phys. 69, 319 (1987)

[26] X. Li, J. Paldus, J. Mol. Struct. (Theochem) 229, 249 (1991)

[27] P. Jordan, Z. Phys 94, 531 (1935)

[28] X. Li, J. Paldus, Theor. Chem. Acc. 133, 1467 (2014)

[29] I. M. Gel'fand, M. L. Tsetlin, Dokl. Akad. Nauk. SSSR 71, 825 (1950) 
[30] H. Weyl, Gruppentheorie und Quantenmechanik, 2nd edn. (Hirzel, Leipzig, 1931); English translation The Theory of Groups and Quantum Mechanics (Dover, New York, 1950, repr. 1964)

[31] A. I. Molev, in Handbook of Algebra, vol.4, eds. by M. Hazewinkel (Elsevier, Amsterdam, 2006) pp. 109-170

[32] J. Paldus, C. R. Sarma, J. Chem. Phys. 83, 5135 (1985)

[33] D. S. Sage, L. Smolinsky, Lithuanian J. Phys. 51, 5 (2011)

[34] J. Paldus, M. J. Boyle, Phys. Scr. 21, 295 (1980)

[35] R. Pauncz, Spin Eigenfunctions: Construction and Use (Plenum, New York, 1979)

[36] S. Wilson, Electron Correlation in Molecules, chap. 5 (Clarendon, Oxford, 1984)

[37] R. McWeeny, Methods in Molecular Quantum Mechanics, 2nd ed., chap. 10 (Academic, New York, 1989)

[38] M. D. Gould, G. S. Chandler, Int. J. Quantum. Chem. 25, 553 (1984)

[39] M. D. Gould, G. S. Chandler, Int. J. Quantum. Chem. 25, 603 (1984)

[40] M. D. Gould, G. S. Chandler, Int. J. Quantum. Chem. 25, 1089 (1984)

[41] M. D. Gould, G. S. Chandler, Int. J. Quantum. Chem. 26, 441 (1984)

[42] M. D. Gould, G. S. Chandler, Int. J. Quantum. Chem. 27, 878 (1984) (Erratum)

[43] R. Shepard, J. Phys. Chem. A. 109, 11629 (2005)

[44] G. Gidofalvi and R. Shepard, Mol. Phys. 108, 2717 (2010)

[45] G. Gidofalvi, S. R. Brozell, R. Shepard, Theor. Chem. Acc. 133, 1512 (2014)

[46] G. E. Baird, L. C. Biedenharn, J. Math. Phys. 4, 1449 (1963) 
[47] L. C. Biedenharn, J. D. Louck, The Racah-Wigner Algebra in Quantum Theory (Addison-Wesley, Reading, 1981)

[48] J. G. Nagel, M. Moshinsky, J. Math. Phys. 6, 682 (1965)

[49] M. Moshinsky, Group Theory and the Many-Body Problem (Gordon and Breach, New York, 1968)

[50] D. P. Zhelobenko, Russian Math. Surveys 17, 1 (1962)

[51] D. P. Zhelobenko, in Representations of Lie Groups and Lie Algebras, ed. by A. A. Kirillov (Akademiai Kiado, 1985), pp. 79-106

[52] Hou Pei-yu, Sci. Sinica 15, 763 (1966)

[53] H. S. Green, J. Math. Phys. 12, 2106 (1971)

[54] M. D. Gould, J. Austral. Math. Soc. B 20, 401 (1978)

[55] M. D. Gould, J. Math. Phys. 21, 444 (1980)

[56] M. D. Gould, J. Math. Phys. 22, 15 (1981)

[57] M. D. Gould, J. Math. Phys. 22, 267 (1981)

[58] G. W. F. Drake, M. Schlesinger, Phys. Rev. A 15, 1990 (1977)

[59] J. Paldus, M. J. Boyle, Phys. Scr. 21, 295 (1980)

[60] J. Paldus, M. J. Boyle, Phys. Rev. A 22, 2299 (1980)

[61] M. J. Boyle, J. Paldus, Phys. Rev. A 22, 2316 (1980)

[62] M. W. Lucht, M. D. Gould, J. Chem. Phys. 103, 5590 (1995)

[63] X. Li, J. Paldus, J. Math. Chem. 4, 295 (1990)

[64] X. Li, J. Paldus, J. Math. Chem. 13, 273 (1993) 
[65] X. Li, J. Paldus, J. Math. Chem. 14, 325 (1990)

[66] B. R. Brooks, W. D. Laidig, P. Saxe, H. F. Schaefer III, J. Chem. Phys. 72, $3837(1980)$

[67] H. Lischka, R. Shepard, F. B. Brown, I. Shavitt, Int. J. Quantum. Chem. Symp. 15, $91(1981)$

[68] R. Shepard, J. Simons, Int. J. Quantum. Chem. Symp. 14, 211 (1980)

[69] R. H. A. Evade, M. A. Robb, Chem. Phys. Lett. 83, 362 (1981)

[70] I. Shavitt, Report to NASA (Batelle Columbus Labs, Columbus, 1979)

[71] X. Li, J. Paldus, J. Chem. Phys. 102, 8059 (1995)

[72] X. Li, J. Paldus, J. Chem. Phys. 102, 8897 (1995)

[73] X. Li, J. Paldus, J. Chem. Phys. 103, 6536 (1995)

[74] X. Li, J. Paldus, J. Chem. Phys. 104, 9555 (1996)

[75] X. Li, J. Paldus. in Recent Advances in Computational Chemistry, vol. 3 (World Scientific, Singapore, 1997), pp. 183-219

[76] X. Li, J. Paldus, Int. J. Quantum. Chem. 70, 65 (1998)

[77] X. Li, J. Paldus, Mol. Phys. 94, 41 (1998)

[78] R. Maitra, D. Sinha, D. Mukherjee, J. Chem. Phys. 137, 024105 (2012); 139, 229903 (2013) (Erratum)

[79] S. Sen, A. Shee, D. Mukherjee, J. Chem. Phys. 137, 074104 (2012)

[80] F. Remacle, R. D. Levin, Chem. Phys. Chem. 2, 20 (2001)

[81] M. D. Gould, J. Paldus, Int. J. Quantum. Chem. 30, 327 (1986) 
[82] M. D. Gould, Int. J. Quantum. Chem. 30, 365 (1986)

[83] J. Paldus, M.-J. Gao, J.-Q. Chen, Phys. Rev. A 35, 3197 (1987)

[84] R. D. Kent, M. Schlesinger, Phys. Rev. A48, 4156 (1993)

[85] P. J. Burton, M. D. Gould, J. Chem. Phys. 96, 5261 (1992)

[86] M. D. Gould, G. S. Chandler, J. Chem. Phys. 90, 3680 (1989)

[87] X. Li, J. Paldus, J. Mol. Struct. (тheоchem) 229, 249 (1991)

[88] X. Li, J. Paldus, Int. J. Quantum. Chem. 41, 117 (1992)

[89] J. Paldus, X. Li, in Group Theory in Physics, AIP Conference Proceedings, no. 266, ed. by A./ Frank, T. H. Seligman, K. B. Wolf (American Institute of Physics, New York, 1992), pp. 159-178

[90] J. Paldus, X. Li, in Symmetries in Science VI: From the Rotation Group to Quantum Algebras, ed. by B. Gruber (Plenum, New York, 1993), pp. 573-592

[91] J. Paldus, J. Planelles, Theor. Chim. Acta 89, 13 (1994)

[92] X. Li, J. Paldus, Chem. Phys. 204, 447 (1996)

[93] X. Li, J. Paldus, Int. J. Quantum. Chem. 60, 513 (1996)

[94] J. Paldus, X. Li, in Pauling Legacy: Modern Modelling of the Chemical Bond; Theoretical and Computational Chemistry Series, vol. 6, chap. 17, ed. by Z. B. Maksić, W. J. Orville-Thomas (Elsevier, Amsterdam, 1999), pp. 481-501

[95] M. D. Gould, J. Paldus, G. S. Chandler, J. Chem. Phys. 93, 4142 (1990)

[96] J. Paldus, M. D. Gould, Theor. Chim. Acta 86, 83 (1992)

[97] R. D. Kent, M. Schlesinger, P. S. Ponnapalli, Phys. Rev. B 31, 1264 (1985)

[98] F. Remacle, R. D. Levin, J. Chem. Phys. 110, 5089 (1999) 
[99] F. Remacle, R. D. Levin, J. Phys. Chem. A 104, 2341 (2000)

[100] X. Li, Q. Zhang, Int. J. Quantum. Chem. 36, 599 (1989)

[101] M. D. Gould, J. Paldus, J. Math. Phys. 28, 2304 (1987)

[102] M. D. Gould, J. Paldus, Phys. Rev. A 34, 804 (1986)

[103] M. D. Gould, J. Paldus, J. Chem. Phys. 92, 7394 (1990)

[104] M. D. Gould, J. Chem. Phys. 99, 5961 (1993)

[105] R. D. Kent, M. Schlesinger, Phys. Rev. A 42, 1155 (1990)

[106] R. D. Kent, M. Schlesinger, I. Shavitt, Int. J. Quantum. Chem. 41, 89 (1990)

[107] J. S. Battle, M. D. Gould, Chem. Phys. Lett. 201, 284 (1993)

[108] M. D. Gould, J. S. Battle, J. Chem. Phys. 98, 8843 (1993)

[109] M. D. Gould, J. S. Battle, J. Chem. Phys. 99, 5983 (1993)

[110] J. P. Burton, M. D. Gould, Int. J. Quantum. Chem. 66, 323 (1998)

[111] J. P. Burton, M. D. Gould, Int. J. Quantum. Chem. 66, 345 (1998)

[112] J. P. Burton, M. D. Gould, Int. J. Quantum. Chem. 66, 365 (1998)

[113] H.-L. Lin, Y.-F. Cao, J. Phys. A Math. Gen. 22, 1509 (1989)

[114] F. A. Matsen, Int. J. Quantum. Chem. 32, 71 (1987)

[115] F. A. Matsen, Int. J. Quantum. Chem. 32, 87 (1987)

[116] F. A. Matsen, Int. J. Quantum. Chem. 32, 105 (1987)

[117] J. Paldus, S. Retrup, C. R. Sarma, J. Mol. Struct. (тheоchem) 199, 85 (1989)

[118] X. Li, J. Paldus, Int. J. Quantum. Chem. 36, 127 (1989) 
[119] R. Nikam, C. R. Sarma, J. Math. Phys. 25, 1199 (1984)

[120] C. R. Sarma, J. Paldus J. Math. Phys. 26, 1140 (1985)

[121] D.-N. Verma, Bull. Amer. Meth. Soc. 74, 160 (1968)

[122] J. Patera, in Proceedings of the 14th ICGTMP (Seoul 1985) (World Sci. Publishing, Singapore, 1986) pp. 289-292

[123] S.-P. Li, R. V. Moody, M. Nicolescu, J. Patera, J. Math. Phys. 27, 668 (1986)

[124] K. N. Raghavan, P. Sankaran, J. Math. Phys. 40, 2190 (1999)

[125] P. Littelmann, J. Pure Appl. Algebra 117/118, 447 (1996)

[126] S. Pošta, M. Havlíček, Acta Polytechnica 53, 450 (2013)

[127] J. Paldus, in Proceedings of NATO ASI on Electrons in Finite and Infinite Structures, ed. by P. Phariseau, I. Scheire (Plenum, New York, 1977), pp. 411429.

[128] W. Heitler, F. London, Z.. Phys. 44, 455 (1927)

[129] L. Pauling, E. B. Wilson, Introduction to Quantum Mechanics (McGraw Hill, New York, 1935)

[130] G. W. Wheland, The Theory of Resonance and Its Application to Organic Chemistry (Wiley, New York, 1944)

[131] G. W. Wheland, Resonance Theory in Organic Chemistry (Wiley, New York, $1955)$

[132] J. K. Syrkin, M. E. Djatkina, Chemical Bond and Molecular Structure (Mir, Moscow, 1946) in Russian; English translation: Structure of Molecules and the Chemical Bond (Interscience, New York, 1950)

[133] A. W. Knapp, Lie Groups Beyond an Introduction (Birkhäuser, Boston, 1996) 
Table 1. Defining inequalities for the exponents $a_{j}$ of the Verma monomials (Vversion) [123] of an irreducible $A_{i} \equiv \mathfrak{s l}(i+1, \mathbb{C})$ or $\mathfrak{s u}(i+1)$ representation with the highest weight $\left[\lambda_{1}, \lambda_{2}, \cdots, \lambda_{i}\right],(i=1,2, \cdots, n)$. The dotted line indicates the last inequality for a given $A_{i}$.

\begin{tabular}{|c|c|c|}
\hline \multicolumn{3}{|c|}{$\begin{array}{l}\left(f_{1}^{a_{N}} f_{2}^{a_{N-1}} \cdots f_{n}^{a_{N-n+1}}\right)\left(f_{1}^{a_{N-n}} \cdots f_{n-1}^{a_{N-2 n+2}}\right) \cdots\left(f_{1}^{a_{3}} f_{2}^{a_{2}}\right) f_{1}^{a_{1}} \\
N=n(n+1) / 2\end{array}$} \\
\hline \multirow[t]{2}{*}{$A_{1}$} & & $0 \leq a_{1} \leq \lambda_{1}$ \\
\hline & & $0 \leq a_{2} \leq \lambda_{2}+a_{1}$ \\
\hline \multirow[t]{3}{*}{$A_{2}$} & . . & $0 \leq a_{3} \leq \min \left\{\lambda_{2}, a_{2}\right\}$ \\
\hline & & $0 \leq a_{4} \leq \lambda_{3}+a_{2}$ \\
\hline & & $0 \leq a_{5} \leq \min \left\{\lambda_{3}+a_{3}, a_{4}\right\}$ \\
\hline \multirow[t]{4}{*}{$A_{3}$} & $\ldots \ldots \ldots$ & $0 \leq a_{6} \leq \min \left\{\lambda_{3}, a_{5}\right\}$ \\
\hline & & $0 \leq a_{7} \leq \lambda_{4}+a_{4}$ \\
\hline & & $0 \leq a_{8} \leq \min \left\{\lambda_{4}+a_{5}, a_{7}\right\}$ \\
\hline & & $0 \leq a_{9} \leq \min \left\{\lambda_{4}+a_{6}, a_{8}\right\}$ \\
\hline$A_{4}$ & $\ldots$ & $0 \leq a_{10} \leq \min \left\{\lambda_{4}, a_{9}\right\}$ \\
\hline \multirow{6}{*}{$A_{n-1}$} & : & \\
\hline & & $0 \leq a_{N-n} \leq \min \left\{\lambda_{n-1}, a_{n(n-1) / 2-1}\right\}$ \\
\hline & & $0 \leq a_{N-n+1} \leq \lambda_{n}+a_{N-2 n+2}$ \\
\hline & & $0 \leq a_{N-n+2} \leq \min \left\{\lambda_{n}+a_{N-2 n+3}, a_{N-n+1}\right\}$ \\
\hline & & $0 \leq a_{N-n+3} \leq \min \left\{\lambda_{n}+a_{N-2 n+4}, a_{N-n+2}\right\}$ \\
\hline & : & $0 \leq a_{N-1} \leq \min \left\{\lambda_{n}+a_{N-n}, a_{N-2}\right\}$ \\
\hline$A_{n}$ & $\ldots \ldots \ldots$ & $0 \leq a_{N} \leq \min \left\{\lambda_{n}, a_{N-1}\right\}$ \\
\hline
\end{tabular}


Table 2. The V-version of the Verma basis for the $\langle 2200\rangle \equiv \Gamma(202)$ irrep of $U(4)$ or $\Lambda_{3}=\left[\begin{array}{lll}0 & 2 & 0\end{array}\right]$ irrep of $A_{3} \equiv \mathfrak{s l}(4, \mathbb{C})$. The sequential numbering $i$ of basis vectors $\left|v_{i}\right\rangle$ corresponds to the scheme of Fig. 1 with the exponents $a_{5} a_{4} a_{3} a_{2}$ listed in the second column. Note that $a_{1}=a_{6}=0$. The third column labeled $w_{i}$ gives the level weight, $w_{i}=\sum a_{j}$, of the un-normalized basis vector $\left|v_{i}\right\rangle \equiv|i\rangle$ as given by the generator sequence $F\left(a_{j}\right) \equiv f_{2}^{a_{5}} f_{3}^{a_{4}} f_{1}^{a_{3}} f_{2}^{a_{2}}$, shown in the fourth column, acting on the HWS $|1\rangle \equiv\left|v_{1}\right\rangle=|1 \overline{1} 2 \overline{2}\rangle$, i.e., $\left|v_{i}\right\rangle=F\left(a_{j}\right)\left|v_{1}\right\rangle$. An alternative representation of $\left|v_{i}\right\rangle$ indicating a generic buildup of the Verma basis is given in the fifth column labeled by $f_{j}|k\rangle$. The explicit form of the un-normalized basis vectors $\left|v_{i}\right\rangle \equiv|i\rangle$ as CSFs (up to a phase) is listed in the sixth column and the next seventh column indicates the ionicity $I$ of that state. The rightmost column labeled $i^{\prime}$ provides the number of an equivalent state obtained via the L-scheme of Table 3. The corresponding VB structures are shown in Fig. 2(a) (see the text for details).

\begin{tabular}{|c|c|c|c|c|c|c|c|}
\hline$i$ & $a_{5} a_{4} a_{3} a_{2}$ & $w_{i}$ & $F\left(a_{j}\right)$ & $f_{j}|k\rangle$ & $\left|v_{i}\right\rangle \equiv|i\rangle$ & $I$ & $i^{\prime}$ \\
\hline 1 & 0000 & 0 & 1 & $|1\rangle$ & $|1 \overline{1} 2 \overline{2}\rangle$ & 2 & 1 \\
\hline 2 & 0001 & 1 & $f_{2}$ & $f_{2}|1\rangle$ & $|1 \overline{1} 2 \overline{\overline{3}}\rangle-|1 \overline{1} \overline{2} 3\rangle$ & 1 & 2 \\
\hline 3 & 0002 & 2 & $f_{2}^{2}$ & $f_{2}|2\rangle$ & $|1 \overline{1} 3 \overline{\overline{3}}\rangle$ & 2 & 3 \\
\hline 4 & 0011 & 2 & $f_{1} f_{2}$ & $f_{1}|2\rangle$ & $|12 \overline{2} \overline{3}\rangle-|\overline{1} 2 \overline{2} 3\rangle$ & 1 & 7 \\
\hline 5 & 0012 & 3 & $f_{1} f_{2}^{2}$ & $f_{1}|3\rangle$ & $|1 \overline{2} 3 \overline{3}\rangle-|\overline{1} 23 \overline{3}\rangle$ & 1 & 8 \\
\hline 6 & 0022 & 4 & $f_{1}^{2} f_{2}^{2}$ & $f_{1}|5\rangle$ & $|2 \overline{2} 3 \overline{3}\rangle$ & 2 & 12 \\
\hline 7 & 0101 & 2 & $f_{3} f_{2}$ & $f_{3}|2\rangle$ & $|1 \overline{1} 2 \overline{\overline{4}}\rangle-|1 \overline{1} \overline{2} 4\rangle$ & 1 & 4 \\
\hline 8 & 0102 & 3 & $f_{3} f_{2}^{2}$ & $f_{3}|3\rangle$ & $|1 \overline{1} 3 \overline{4}\rangle-|1 \overline{1} \overline{3} 4\rangle$ & 1 & 5 \\
\hline 9 & 0111 & 3 & $f_{3} f_{1} f_{2}$ & $f_{3}|4\rangle$ & $|12 \overline{2} \overline{4}\rangle-|\overline{1} 2 \overline{2} 4\rangle$ & 1 & 9 \\
\hline 10 & 0112 & 4 & $f_{3} f_{1} f_{2}^{2}$ & $f_{3}|5\rangle$ & $|1 \overline{2} 3 \overline{4}\rangle-|1 \overline{2} \overline{3} 4\rangle-|\overline{1} 23 \overline{4}\rangle+|\overline{1} 2 \overline{3} 4\rangle$ & 0 & 10 \\
\hline 11 & 0122 & 5 & $f_{3} f_{1}^{2} f_{2}^{2}$ & $f_{3}|6\rangle$ & $|2 \overline{2} 3 \overline{4}\rangle-|2 \overline{2} \overline{3} 4\rangle$ & 1 & 13 \\
\hline 12 & 0202 & 4 & $f_{3}^{2} f_{2}^{2}$ & $f_{3}|8\rangle$ & $|1 \overline{1} 4 \overline{4}\rangle$ & 2 & 6 \\
\hline 13 & 0212 & 5 & $f_{3}^{2} f_{1} f_{2}^{2}$ & $f_{3}|10\rangle$ & $|1 \overline{2} 4 \overline{\overline{4}}\rangle-|\overline{1} 24 \overline{4}\rangle$ & 1 & 11 \\
\hline 14 & 0222 & 6 & $f_{3}^{2} f_{1}^{2} f_{2}^{2}$ & $f_{3}|11\rangle$ & $|2 \overline{2} 4 \overline{\overline{4}}\rangle$ & 2 & 14 \\
\hline 15 & 1111 & 4 & $f_{2} f_{3} f_{1} f_{2}$ & $f_{2}|9\rangle$ & $|12 \overline{3} \overline{4}\rangle-|1 \overline{2} 3 \overline{\overline{4}}\rangle-|\overline{1} 2 \overline{3} 4\rangle+|\overline{1} \overline{2} 34\rangle$ & 0 & 15 \\
\hline 16 & 1112 & 5 & $f_{2} f_{3} f_{1} f_{2}^{2}$ & $f_{2}|10\rangle$ & $|13 \overline{3} \overline{4}\rangle-|\overline{1} 3 \overline{3} 4\rangle$ & 1 & 16 \\
\hline 17 & 1122 & 6 & $f_{2} f_{3} f_{1}^{2} f_{2}^{2}$ & $f_{2}|11\rangle$ & $|23 \overline{3} \overline{4}\rangle-|\overline{2} 3 \overline{3} \overline{4}\rangle$ & 1 & 18 \\
\hline 18 & 1212 & 6 & $f_{2} f_{3}^{2} f_{1} f_{2}^{2}$ & $f_{2}|13\rangle$ & $|1 \overline{3} 4 \overline{4}\rangle-|\overline{1} 34 \overline{4}\rangle$ & 1 & 17 \\
\hline 19 & 1222 & 7 & $f_{2} f_{3}^{2} f_{1}^{2} f_{2}^{2}$ & $f_{2}|14\rangle$ & $|2 \overline{3} 4 \overline{4}\rangle-|\overline{2} 34 \overline{4}\rangle$ & 1 & 19 \\
\hline 20 & 2222 & 8 & $f_{2}^{2} f_{3}^{2} f_{1}^{2} f_{2}^{2}$ & $f_{2}|19\rangle$ & $|3 \overline{3} 4 \overline{\overline{4}}\rangle$ & 2 & 20 \\
\hline
\end{tabular}


Table 3. The L-version of the Verma basis for the $\langle 2200\rangle \equiv \Gamma(202)$ irrep of U(4) or $\Lambda_{3}=\left[\begin{array}{lll}0 & 2 & 0\end{array}\right]$ irrep of $A_{3} \equiv \mathfrak{s l}(4, \mathbb{C})$. The sequential numbering $i$ of basis vectors $\left|v_{i}\right\rangle$ corresponds to the scheme of Fig. 1 with $a_{2}, a_{3}, a_{4}$, and $a_{5}$ replaced by $a_{2}^{3}, a_{3}^{3}, a_{1}^{2}$, and $a_{2}^{2}$ (listed in the second column), respectively, as in Table 2. Note that $a_{1}^{1}=a_{1}^{3}=0$. The third column gives level weight $w_{i}, w_{i}=\sum a_{k}^{c}$, of the un-normalized basis vector $\left|v_{i}\right\rangle \equiv|i\rangle$ given by the generator sequence $F\left(a_{k}^{c}\right) \equiv f_{2}^{a_{2}^{2}} f_{1}^{a_{1}^{2}} f_{3}^{a_{3}^{3}} f_{2}^{a_{2}^{3}}$ (shown in the fourth column) acting on the HWS $\left|v_{1}\right\rangle=|1 \overline{1} 2 \overline{2}\rangle$, i.e., $\left|v_{i}\right\rangle=F\left(a_{k}^{c}\right)\left|v_{1}\right\rangle$. An alternative representation of $\left|v_{i}\right\rangle$ indicating the generic buildup of the Verma basis is given in the fifth column labeled by $f_{j}|k\rangle$. The explicit form of the un-normalized basis vectors $\left|v_{i}\right\rangle \equiv|i\rangle$ as CSFs (up to a phase) is listed in the sixth column and the next seventh column indicates the ionicity $I$ of that state. In the rightmost column labeled $i^{\prime}$ we provide the number of an equivalent state from Table 2.

\begin{tabular}{|c|c|c|c|c|c|c|c|}
\hline$i$ & $a_{2}^{2} a_{1}^{2} a_{3}^{3} a_{2}^{3}$ & $w_{i}$ & $F\left(a_{k}^{c}\right)$ & $f_{j}|k\rangle$ & $\left|v_{i}\right\rangle \equiv|i\rangle$ & $I$ & $i^{\prime}$ \\
\hline 1 & 0000 & 0 & 1 & $|1\rangle$ & $|1 \overline{1} 2 \overline{2}\rangle$ & 2 & 1 \\
\hline 2 & 0001 & 1 & $f_{2}$ & $f_{2}|1\rangle$ & $|1 \overline{1} 2 \overline{3}\rangle-|1 \overline{1} \overline{2} 3\rangle$ & 1 & 2 \\
\hline 3 & 0002 & 2 & $f_{2}^{2}$ & $f_{2}|2\rangle$ & $|1 \overline{1} 3 \overline{3}\rangle$ & 2 & 3 \\
\hline 4 & 0011 & 2 & $f_{3} f_{2}$ & $f_{3}|2\rangle$ & $|1 \overline{1} 2 \overline{4}\rangle-|1 \overline{1} \overline{2} 4\rangle$ & 1 & 7 \\
\hline 5 & 0012 & 3 & $f_{3} f_{2}^{2}$ & $f_{3}|3\rangle$ & $|1 \overline{1} 3 \overline{4}\rangle-|1 \overline{1} \overline{3} 4\rangle$ & 1 & 8 \\
\hline 6 & 0022 & 4 & $f_{3}^{2} f_{2}^{2}$ & $f_{3}|5\rangle$ & $|1 \overline{1} 4 \overline{\overline{4}}\rangle$ & 2 & 12 \\
\hline 7 & 0101 & 2 & $f_{1} f_{2}$ & $f_{1}|2\rangle$ & $|12 \overline{2} \overline{\overline{3}}\rangle-|\overline{1} 2 \overline{2} 3\rangle$ & 2 & 4 \\
\hline 8 & 0102 & 3 & $f_{1} f_{2}^{2}$ & $f_{1}|3\rangle$ & $|1 \overline{2} 3 \overline{3}\rangle-|\overline{1} 23 \overline{3}\rangle$ & 1 & 5 \\
\hline 9 & 0111 & 3 & $f_{1} f_{3} f_{2}$ & $f_{1}|4\rangle$ & $|12 \overline{2} \overline{4}\rangle-|\overline{1} 2 \overline{2} 4\rangle$ & 1 & 9 \\
\hline 10 & 0112 & 4 & $f_{1} f_{3} f_{2}^{2}$ & $f_{1}|5\rangle$ & $|1 \overline{2} 3 \overline{4}\rangle-|1 \overline{2} \overline{3} 4\rangle-|\overline{1} 23 \overline{4}\rangle+|\overline{1} 2 \overline{3} 4\rangle$ & 0 & 10 \\
\hline 11 & 0122 & 5 & $f_{1} f_{3}^{2} f_{2}^{2}$ & $f_{1}|6\rangle$ & $|1 \overline{2} 4 \overline{4}\rangle-|\overline{1} 24 \overline{4}\rangle$ & 1 & 13 \\
\hline 12 & 0202 & 4 & $f_{1}^{2} f_{2}^{2}$ & $f_{1}|8\rangle$ & $|2 \overline{2} 3 \overline{3}\rangle$ & 2 & 6 \\
\hline 13 & 0212 & 5 & $f_{1}^{2} f_{3} f_{2}^{2}$ & $f_{1}|10\rangle$ & $|2 \overline{2} 3 \overline{\overline{4}}\rangle-|2 \overline{2} \overline{3} 4\rangle$ & 1 & 11 \\
\hline 14 & 0222 & 6 & $f_{1}^{2} f_{3}^{2} f_{2}^{2}$ & $f_{1}|11\rangle$ & $|2 \overline{2} 4 \overline{\overline{4}}\rangle$ & 2 & 14 \\
\hline 15 & 1111 & 4 & $f_{2} f_{1} f_{3} f_{2}$ & $f_{2}|9\rangle$ & $|12 \overline{3} \overline{4}\rangle-|1 \overline{2} 3 \overline{4}\rangle-|\overline{1} 2 \overline{3} 4\rangle+|\overline{1} \overline{2} 34\rangle$ & 0 & 15 \\
\hline 16 & 1112 & 5 & $f_{2} f_{1} f_{3} f_{2}^{2}$ & $f_{2}|10\rangle$ & $|13 \overline{3} \overline{4}\rangle-|\overline{1} 3 \overline{3} 4\rangle$ & 1 & 16 \\
\hline 17 & 1122 & 6 & $f_{2} f_{1} f_{3}^{2} f_{2}^{2}$ & $f_{2}|11\rangle$ & $|1 \overline{3} 4 \overline{4}\rangle-|\overline{1} 34 \overline{4}\rangle$ & 1 & 18 \\
\hline 18 & 1212 & 6 & $f_{2} f_{1}^{2} f_{3} f_{2}^{2}$ & $f_{2}|13\rangle$ & $|23 \overline{3} \overline{4}\rangle-|\overline{2} 3 \overline{3} 4\rangle$ & 1 & 17 \\
\hline 19 & 1222 & 7 & $f_{2} f_{1}^{2} f_{3}^{2} f_{2}^{2}$ & $f_{2}|14\rangle$ & $|2 \overline{3} 4 \overline{4}\rangle-|\overline{2} 34 \overline{4}\rangle$ & 1 & 19 \\
\hline 20 & 2222 & 8 & $f_{2}^{2} f_{1}^{2} f_{3}^{2} f_{2}^{2}$ & $f_{2}|19\rangle$ & $|3 \overline{3} 4 \overline{4}\rangle$ & 2 & 20 \\
\hline
\end{tabular}


Table 4. The V-version of the Verma basis for the $\langle 2110\rangle \equiv \Gamma(121)$ irrep of $\mathrm{U}(4)$ or $\Lambda_{3}=\left[\begin{array}{lll}1 & 0 & 1\end{array}\right]$ irrep of $A_{3} \equiv \mathfrak{s l}(4, \mathbb{C})$. The sequential numbering $i$ of basis vectors $\left|v_{i}\right\rangle$ corresponds to the scheme of Fig. 4 with the exponents $a_{6} a_{5} a_{4} a_{2} a_{1}$ listed in the second column. Note that $a_{3}=0$. The level weight $w_{i}, w_{i}=\sum a_{k}^{c}$ (shown in the third column) of the un-normalized basis vectors $\left|v_{i}\right\rangle$ (up to a phase) as given by the generator sequence $F\left(a_{j}\right) \equiv f_{1}^{a_{6}} f_{2}^{a_{5}} f_{3}^{a_{4}} f_{2}^{a_{2}} f_{1}^{a_{1}}$ (shown in the fourth column) acting on the HWS $\left|v_{1}\right\rangle=|1 \overline{1} 23\rangle$, i.e., $\left|v_{i}\right\rangle=F\left(a_{j}\right)\left|v_{1}\right\rangle$, are listed in the rightmost column. An alternative representation of $\left|v_{i}\right\rangle$ indicating the generic buildup of the Verma basis is given in the fifth column labeled by $f_{j}|k\rangle$. The corresponding VB structures are shown in Fig. 6 (see the text for details).

\begin{tabular}{rcrrrc}
\hline \hline$i$ & $a_{6} a_{5} a_{4} a_{2} a_{1}$ & $w_{i}$ & $F\left(a_{j}\right)$ & $f_{j}|k\rangle$ & $\left|v_{i}\right\rangle=|i\rangle$ \\
\hline 1 & 00000 & 0 & 1 & $|1\rangle$ & $|1 \overline{1} 23\rangle$ \\
2 & 00001 & 1 & $f_{1}$ & $f_{1}|1\rangle$ & $|12 \overline{2} 3\rangle$ \\
3 & 00011 & 2 & $f_{2} f_{1}$ & $f_{2}|2\rangle$ & $|123 \overline{3}\rangle$ \\
4 & 00100 & 1 & $f_{3}$ & $f_{3}|1\rangle$ & $|1 \overline{1} 24\rangle$ \\
5 & 00101 & 2 & $f_{3} f_{1}$ & $f_{3}|2\rangle$ & $|12 \overline{2} 4\rangle$ \\
6 & 00111 & 3 & $f_{3} f_{2} f_{1}$ & $f_{3}|3\rangle$ & $|123 \overline{4}\rangle-|12 \overline{3} 4\rangle$ \\
7 & 00211 & 4 & $f_{3}^{2} f_{2} f_{1}$ & $f_{3}|6\rangle$ & $|124 \overline{4}\rangle$ \\
8 & 01100 & 2 & $f_{2} f_{3}$ & $f_{2}|4\rangle$ & $|1 \overline{1} 34\rangle$ \\
9 & 01101 & 3 & $f_{2} f_{3} f_{1}$ & $f_{2}|5\rangle$ & $|12 \overline{3} 4\rangle-|1 \overline{2} 34\rangle$ \\
10 & 01111 & 4 & $f_{2} f_{3} f_{2} f_{1}$ & $f_{2}|6\rangle$ & $|13 \overline{3} 4\rangle$ \\
11 & 01211 & 5 & $f_{2} f_{3}^{2} f_{2} f_{1}$ & $f_{2}|7\rangle$ & $|134 \overline{4}\rangle$ \\
12 & 11100 & 3 & $f_{1} f_{2} f_{3}$ & $f_{1}|8\rangle$ & $|1 \overline{2} 34\rangle-|\overline{1} 234\rangle$ \\
13 & 11101 & 4 & $f_{1} f_{2} f_{3} f_{1}$ & $f_{1}|9\rangle$ & $|2 \overline{2} 34\rangle$ \\
14 & 11111 & 5 & $f_{1} f_{2} f_{3} f_{2} f_{1}$ & $f_{1}|10\rangle$ & $|23 \overline{3} 4\rangle$ \\
15 & 11211 & 6 & $f_{1} f_{2} f_{3}^{2} f_{2} f_{1}$ & $f_{1}|11\rangle$ & $|234 \overline{4}\rangle$ \\
\hline \hline
\end{tabular}


Table 5. The V-version of the Verma basis for the $\langle 2100\rangle \equiv \Gamma(112)$ irrep of $\mathrm{U}(4)$ or $\Lambda_{3}=\left[\begin{array}{lll}1 & 1 & 0\end{array}\right]$ irrep of $A_{3} \equiv \mathfrak{s l}(4, \mathbb{C})$. The sequential numbering $i$ of basis vectors $\left|v_{i}\right\rangle$ corresponds to the lexical order of exponents $a_{i},(i=1, \cdots, 5)$ listed in the second column and obtained in a similar way as shown in Fig. 1 for the [020] irrep. Note that $a_{6}=0$. The level weight $w_{i}, w_{i}=\sum a_{j}$ are listed in the third column and the un-normalized basis vectors $\left|v_{i}\right\rangle$ (up to a phase) as given by the generator sequence $F\left(a_{j}\right) \equiv f_{2}^{a_{5}} f_{3}^{a_{4}} f_{1}^{a_{3}} f_{2}^{a_{2}} f_{1}^{a_{1}}$ (shown in the fourth column) acting on the HWS $\left|v_{1}\right\rangle=|1 \overline{1} 2\rangle$, i.e., $\left|v_{i}\right\rangle=F\left(a_{j}\right)\left|v_{1}\right\rangle$, are listed in the rightmost column. An alternative representation of $\left|v_{i}\right\rangle$ indicating the generic buildup of the Verma basis is given in the fifth column labeled by $f_{j}|k\rangle$.

\begin{tabular}{rcrrcc}
\hline \hline$i$ & $a_{5} a_{4} a_{3} a_{2} a_{1}$ & $w_{i}$ & $F\left(a_{i}\right)$ & $f_{j}|k\rangle$ & $\left|v_{i}\right\rangle \equiv|i\rangle$ \\
\hline 1 & 00000 & 0 & 1 & $|1\rangle$ & $|1 \overline{1} 2\rangle$ \\
2 & 00001 & 1 & $f_{1}$ & $f_{1}|1\rangle$ & $|12 \overline{2}\rangle$ \\
3 & 00010 & 1 & $f_{2}$ & $f_{2}|1\rangle$ & $|1 \overline{1} 3\rangle$ \\
4 & 00011 & 2 & $f_{2} f_{1}$ & $f_{2}|2\rangle$ & $|12 \overline{3}\rangle-|1 \overline{2} 3\rangle$ \\
5 & 00021 & 3 & $f_{2}^{2} f_{1}$ & $f_{2}|4\rangle$ & $|13 \overline{3}\rangle$ \\
6 & 00110 & 2 & $f_{1} f_{2}$ & $f_{1}|3\rangle$ & $|1 \overline{2} 3\rangle-|\overline{1} 23\rangle$ \\
7 & 00111 & 3 & $f_{1} f_{2} f_{1}$ & $f_{1}|4\rangle$ & $|2 \overline{2} 3\rangle$ \\
8 & 00121 & 4 & $f_{1} f_{2}^{2} f_{1}$ & $f_{1}|5\rangle$ & $|23 \overline{3}\rangle$ \\
9 & 01010 & 2 & $f_{3} f_{2}$ & $f_{3}|3\rangle$ & $|1 \overline{1} 4\rangle$ \\
10 & 01011 & 3 & $f_{3} f_{2} f_{1}$ & $f_{3}|4\rangle$ & $|12 \overline{4}\rangle-|1 \overline{2} 4\rangle$ \\
11 & 01021 & 4 & $f_{3} f_{2}^{2} f_{1}$ & $f_{3}|5\rangle$ & $|13 \overline{4}\rangle-|1 \overline{3} 4\rangle$ \\
12 & 01110 & 3 & $f_{3} f_{1} f_{2}$ & $f_{3}|6\rangle$ & $|1 \overline{2} 4\rangle-|\overline{1} 24\rangle$ \\
13 & 01111 & 4 & $f_{3} f_{1} f_{2} f_{1}$ & $f_{3}|7\rangle$ & $|2 \overline{2} 4\rangle$ \\
14 & 01121 & 5 & $f_{3} f_{1} f_{2}^{2} f_{1}$ & $f_{3}|8\rangle$ & $|23 \overline{4}\rangle-|2 \overline{3} 4\rangle$ \\
15 & 02021 & 5 & $f_{3}^{2} f_{2}^{2} f_{1}$ & $f_{3}|11\rangle$ & $|14 \overline{4}\rangle$ \\
16 & 02121 & 6 & $f_{3}^{2} f_{1} f_{2}^{2} f_{1}$ & $f_{3}|14\rangle$ & $|24 \overline{4}\rangle$ \\
17 & 11110 & 4 & $f_{2} f_{3} f_{1} f_{2}$ & $f_{2}|12\rangle$ & $|1 \overline{3} 4\rangle-|\overline{1} 34\rangle$ \\
18 & 11111 & 5 & $f_{2} f_{3} f_{1} f_{2} f_{1}$ & $f_{2}|13\rangle$ & $|2 \overline{3} 4\rangle-|\overline{2} 34\rangle$ \\
19 & 11121 & 6 & $f_{2} f_{3} f_{1} f_{2}^{2} f_{1}$ & $f_{2}|14\rangle$ & $|3 \overline{3} 4\rangle$ \\
20 & 12121 & 7 & $f_{2} f_{3}^{2} f_{1} f_{2}^{2} f_{1}$ & $f_{2}|16\rangle$ & $|34 \overline{4}\rangle$ \\
\hline \hline
\end{tabular}


Table 6. The V-version of the Verma basis for the $\langle 2000\rangle \equiv \Gamma(103)$ irrep of $\mathrm{U}(4)$ or $\Lambda_{3}=\left[\begin{array}{lll}2 & 0 & 0\end{array}\right]$ irrep of $A_{3} \equiv \mathfrak{s l}(4, \mathbb{C})$. The sequential numbering $i$ of basis vectors $\left|v_{i}\right\rangle$ corresponds to the lexical order of exponents $a_{4}, a_{2}$ and $a_{1}$, listed in the second column. Note that $a_{3}=a_{5}=a_{6}=0$. The level weight $w_{i}, w_{i}=\sum a_{j}$ (shown in the third column) of the un-normalized basis vectors $\left|v_{i}\right\rangle$ (up to a phase), as given by the generator sequence $F\left(a_{j}\right) \equiv f_{3}^{a_{4}} f_{2}^{a_{2}} f_{1}^{a_{1}}$ (shown in the fourth column) acting on the HWS $\left|v_{1}\right\rangle=|1 \overline{1}\rangle$, i.e., $\left|v_{i}\right\rangle=F\left(a_{j}\right)\left|v_{1}\right\rangle$, are listed in the rightmost column. An alternative representation of $\left|v_{i}\right\rangle$ indicating the generic buildup of the Verma basis is given in the fifth column labeled by $f_{j}|k\rangle$.

\begin{tabular}{rcrrrc}
\hline \hline$i$ & $a_{4} a_{2} a_{1}$ & $w_{i}$ & $F\left(a_{i}\right)$ & $f_{j}|k\rangle$ & $\left|v_{i}\right\rangle \equiv|i\rangle$ \\
\hline 1 & 000 & 0 & 1 & $|1\rangle$ & $|1 \overline{1}\rangle$ \\
2 & 001 & 1 & $f_{1}$ & $f_{1}|1\rangle$ & $|1 \overline{2}\rangle-|\overline{1} 2\rangle$ \\
3 & 002 & 2 & $f_{1}^{2}$ & $f_{1}|2\rangle$ & $|2 \overline{2}\rangle$ \\
4 & 011 & 2 & $f_{2} f_{1}$ & $f_{2}|2\rangle$ & $|1 \overline{3}\rangle-|\overline{1} 3\rangle$ \\
5 & 012 & 3 & $f_{2} f_{1}^{2}$ & $f_{2}|3\rangle$ & $|2 \overline{3}\rangle-|\overline{2} 3\rangle$ \\
6 & 022 & 4 & $f_{2}^{2} f_{1}^{2}$ & $f_{2}|5\rangle$ & $|3 \overline{3}\rangle$ \\
7 & 111 & 3 & $f_{3} f_{2} f_{1}$ & $f_{3}|4\rangle$ & $|1 \overline{4}\rangle-|\overline{1} 4\rangle$ \\
8 & 112 & 4 & $f_{2} f_{2} f_{1}^{2}$ & $f_{3}|5\rangle$ & $|2 \overline{4}\rangle-|\overline{2} 4\rangle$ \\
9 & 122 & 5 & $f_{3} f_{2}^{2} f_{1}^{2}$ & $f_{3}|6\rangle$ & $|3 \overline{4}\rangle-|\overline{3} 4\rangle$ \\
10 & 222 & 6 & $f_{3}^{2} f_{2}^{2} f_{1}^{2}$ & $f_{3}|9\rangle$ & $|4 \overline{4}\rangle$ \\
\hline \hline
\end{tabular}


Table 7. The V-version of the Verma basis for the $\langle 200000\rangle \equiv\langle 2 \dot{0}\rangle \equiv \Gamma(105)$ irrep of $\mathrm{U}(6)$ or $\Lambda_{5}=[2 \dot{0}] \equiv[2]$ irrep of $A_{5} \equiv \mathfrak{s l}(5, \mathbb{C})$. The sequential numbering $i$ of basis vectors $\left|v_{i}\right\rangle$ corresponds to the lexical order of exponents $a_{11}, a_{7}, a_{4}, a_{2}$ and $a_{1}$, listed in the second column. Note that $a_{i}=0$, for $i=3,5,6,8-10,12-15$. The level weight $w_{i}, w_{i}=\sum a_{j}$ (shown in the third column) of the un-normalized basis vectors $\left|v_{i}\right\rangle$ (up to a phase), as given by the generator sequence $F\left(a_{j}\right) \equiv f_{5}^{a_{11}} f_{4}^{a_{7}} f_{3}^{a_{4}} f_{2}^{a_{2}} f_{1}^{a_{1}}$ (shown in the fourth column) acting on the HWS $\left|v_{1}\right\rangle=|1 \overline{1}\rangle$, i.e., $\left|v_{i}\right\rangle=F\left(a_{j}\right)\left|v_{1}\right\rangle$, are listed in the rightmost column. An alternative representation of $\left|v_{i}\right\rangle$ indicating the generic buildup of the Verma basis is given in the fifth column labeled by $f_{j}|k\rangle$.

\begin{tabular}{rrrrrc}
\hline \hline$i$ & $a_{11} a_{7} a_{4} a_{2} a_{1}$ & $w_{i}$ & $F\left(a_{j}\right)$ & $f_{j}|k\rangle$ & $\left|v_{i}\right\rangle \equiv|i\rangle$ \\
\hline 1 & 00000 & 0 & 1 & $|1\rangle$ & $|1 \overline{1}\rangle$ \\
2 & 00001 & 1 & $f_{1}$ & $f_{1}|1\rangle$ & $|1 \overline{2}\rangle-|\overline{1} 2\rangle$ \\
3 & 00002 & 2 & $f_{1}^{2}$ & $f_{1}|2\rangle$ & $|2 \overline{2}\rangle$ \\
4 & 00011 & 2 & $f_{2} f_{1}$ & $f_{2}|2\rangle$ & $|1 \overline{3}\rangle-|\overline{1} 3\rangle$ \\
5 & 00012 & 3 & $f_{2} f_{1}^{2}$ & $f_{2}|3\rangle$ & $|2 \overline{3}\rangle-|\overline{2} 3\rangle$ \\
6 & 00022 & 4 & $f_{2}^{2} f_{1}^{2}$ & $f_{2}|5\rangle$ & $|3 \overline{3}\rangle$ \\
7 & 00111 & 3 & $f_{3} f_{2} f_{1}$ & $f_{3}|4\rangle$ & $|1 \overline{4}\rangle-|\overline{1} 4\rangle$ \\
8 & 00112 & 4 & $f_{3} f_{2} f_{1}^{2}$ & $f_{3}|5\rangle$ & $|2 \overline{4}\rangle-|\overline{2} 4\rangle$ \\
9 & 00122 & 5 & $f_{3} f_{2}^{2} f_{1}^{2}$ & $f_{3}|6\rangle$ & $|3 \overline{4}\rangle-|\overline{3} 4\rangle$ \\
10 & 00222 & 6 & $f_{3}^{2} f_{2}^{2} f_{1}^{2}$ & $f_{3}|9\rangle$ & $|4 \overline{4}\rangle$ \\
11 & 01111 & 4 & $f_{4} f_{3} f_{2} f_{1}$ & $f_{4}|7\rangle$ & $|1 \overline{5}\rangle-|\overline{1} 5\rangle$ \\
12 & 01112 & 5 & $f_{4} f_{3} f_{2} f_{1}^{2}$ & $f_{4}|8\rangle$ & $|2 \overline{5}\rangle-|\overline{2} 5\rangle$ \\
13 & 01122 & 6 & $f_{4} f_{3} f_{2}^{2} f_{1}^{2}$ & $f_{4}|9\rangle$ & $|3 \overline{5}\rangle-|\overline{3} 5\rangle$ \\
14 & 01222 & 7 & $f_{4} f_{3}^{2} f_{2}^{2} f_{1}^{2}$ & $f_{4}|10\rangle$ & $|4 \overline{5}\rangle-|\overline{4} 5\rangle$ \\
15 & 02222 & 8 & $f_{4}^{2} f_{3}^{2} f_{2}^{2} f_{1}^{2}$ & $f_{4}|14\rangle$ & $|5 \overline{5}\rangle$ \\
16 & 11111 & 5 & $f_{5} f_{4} f_{3} f_{2} f_{1}$ & $f_{5}|11\rangle$ & $|1 \overline{6}\rangle-|\overline{1} 6\rangle$ \\
17 & 11112 & 6 & $f_{5} f_{4} f_{3} f_{2} f_{1}^{2}$ & $f_{5}|12\rangle$ & $|2 \overline{6}\rangle-|\overline{2} 6\rangle$ \\
18 & 11122 & 7 & $f_{5} f_{4} f_{3} f_{2}^{2} f_{1}^{2}$ & $f_{5}|13\rangle$ & $|3 \overline{6}\rangle-|\overline{3} 6\rangle$ \\
19 & 11222 & 8 & $f_{5} f_{4} f_{3}^{2} f_{2}^{2} f_{1}^{2}$ & $f_{5}|14\rangle$ & $|4 \overline{6}\rangle-|\overline{4} 6\rangle$ \\
20 & 12222 & 9 & $f_{5} f_{4}^{2} f_{3}^{2} f_{2}^{2} f_{1}^{2}$ & $f_{5}|15\rangle$ & $|5 \overline{6}\rangle-|\overline{5} 6\rangle$ \\
21 & 22222 & 10 & $f_{5}^{2} f_{4}^{2} f_{3}^{2} f_{2}^{2} f_{1}^{2}$ & $f_{5}|20\rangle$ & $|6 \overline{6}\rangle$ \\
\hline \hline
\end{tabular}




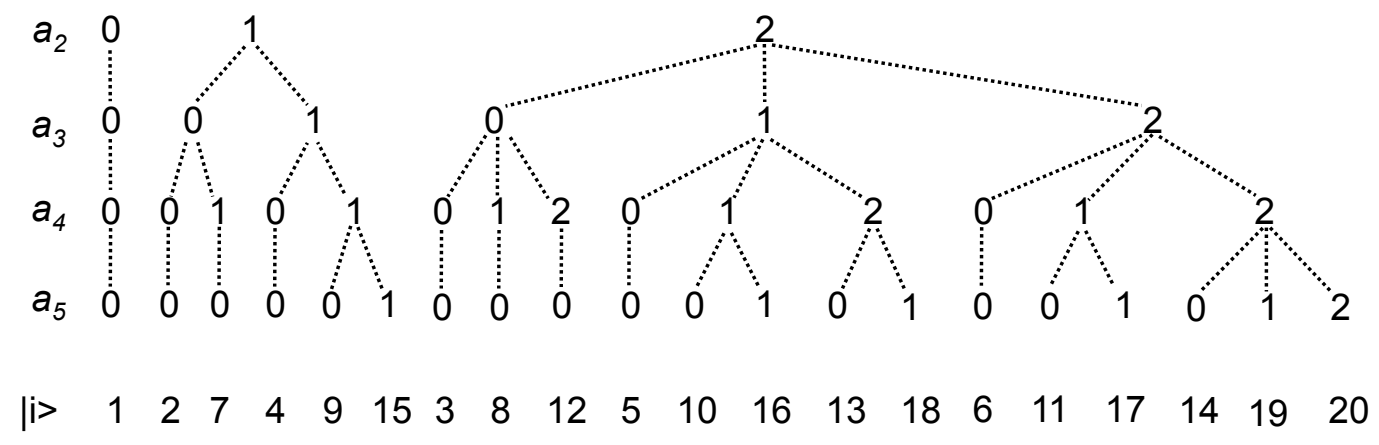

Fig. 1 List of non-vanishing exponents $a_{i}, i=2, \cdots, 5$ generating Verma monomials $f_{2}^{a_{5}} f_{3}^{a_{4}} f_{1}^{a_{3}} f_{2}^{a_{2}}$ for the $A_{3}$ irrep $\Lambda_{3}=\left[\begin{array}{ll}0 & 2\end{array}\right]$, arranged in a natural generating order. The implied lexical numbering of CSFs $|i\rangle \equiv\left|v_{i}\right\rangle, i=1, \cdots, 20$ is given in the bottom row. The same diagram applies to the irrep $\Lambda_{3}=\left[\begin{array}{lll}1 & 0 & 1\end{array}\right]$ when we replace exponents $a_{2} a_{3} a_{4} a_{5}$ by $a_{2}^{3} a_{3}^{3} a_{1}^{2} a_{2}^{2}$ and $f_{2}^{a_{5}} f_{3}^{a_{4}} f_{1}^{a_{3}} f_{2}^{a_{2}}$ by $f_{2}^{a_{2}^{2}} f_{1}^{a_{1}^{2}} f_{3}^{a_{3}^{3}} f_{2}^{a_{2}^{3}}$, respectively. 
(a)

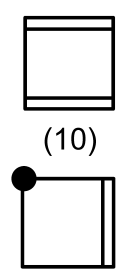

(2)

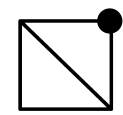

(4)

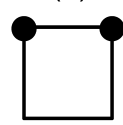

(1)

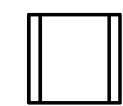

(15)

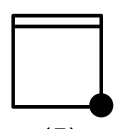

(5)

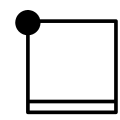

(8)

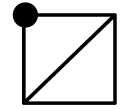

(7)

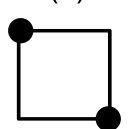

(3)

(17)

(6)
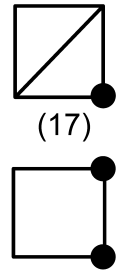

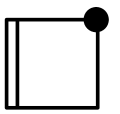

(9)

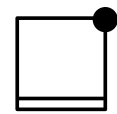

(11)

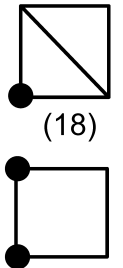

(12)

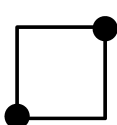

(14)

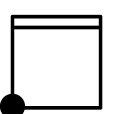

(13)

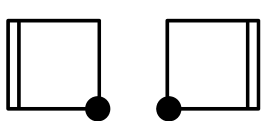

(16)

(19)

(b) $\square_{4}^{1}$

Fig. 2 (a) Valence bond structures representing the Verma basis vectors (or CSFs)

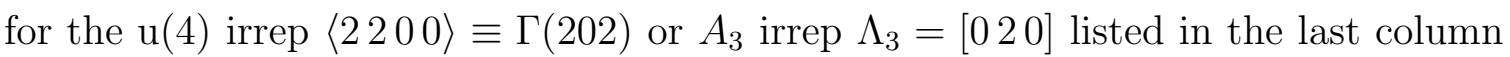
of Table 2, applied to the MBS PPP model of cyclobutadiene. The two valence structures are in the 1st row, the 12 singly-ionic structure are in the next two rows and the six doubly-ionic structures are in the bottom row. The ionicity $I, I=0,1,2$, is given by the number of dots indicating a double occupancy of the site. (b) The numbering of the atomic sites used in part (a). 

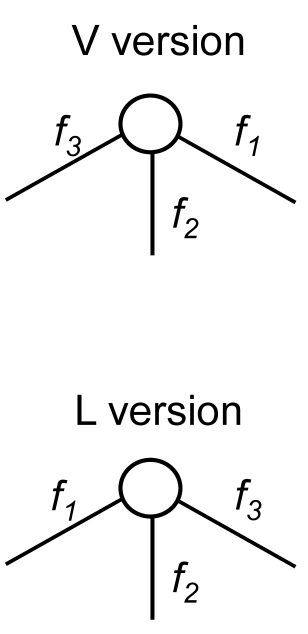

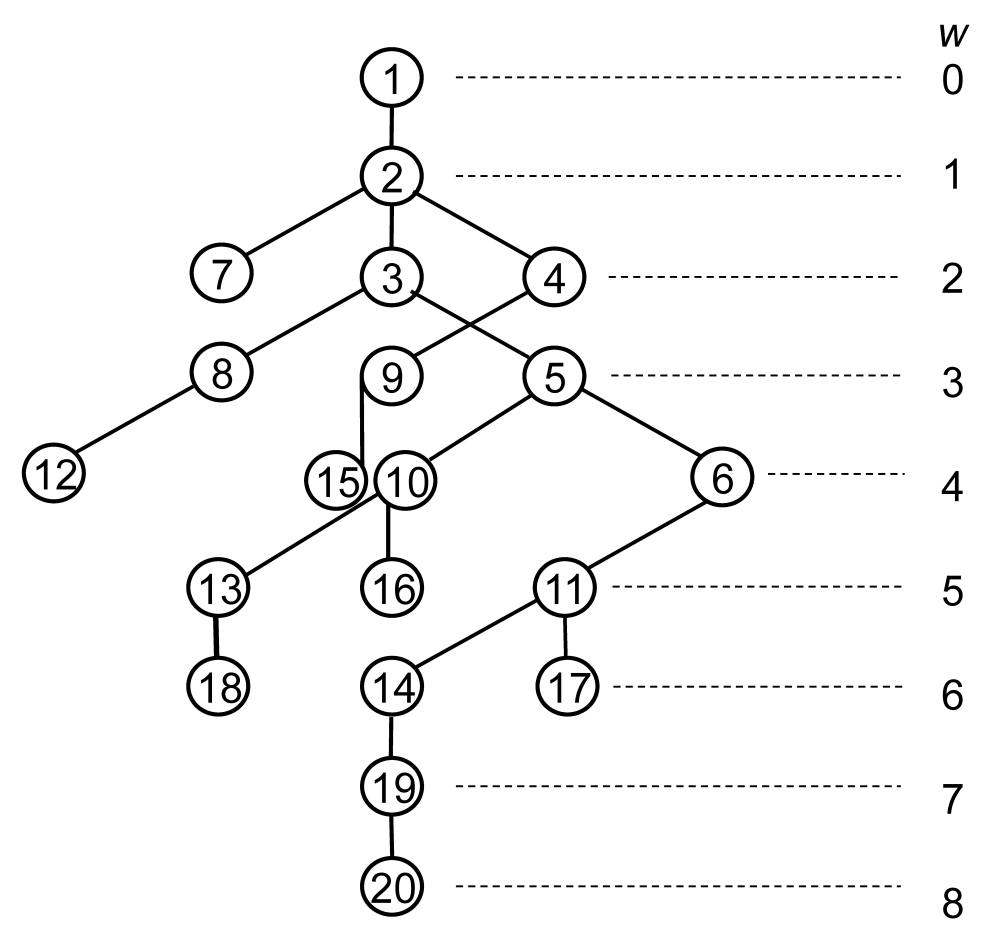

Fig. 3 The HLED diagram for the Verma basis of the U(4) irrep $\langle 2200\rangle \equiv \Gamma(202)$ or $A_{3} \equiv \mathfrak{s l}(4, \mathbb{C})$ irrep $\Lambda_{3}=\left[\begin{array}{lll}0 & 2 & 0\end{array}\right]$ of Table 2 (V-version) and Table 3 (L-version). The level weight $w_{i} \equiv w$ is indicated on the right hand side. 

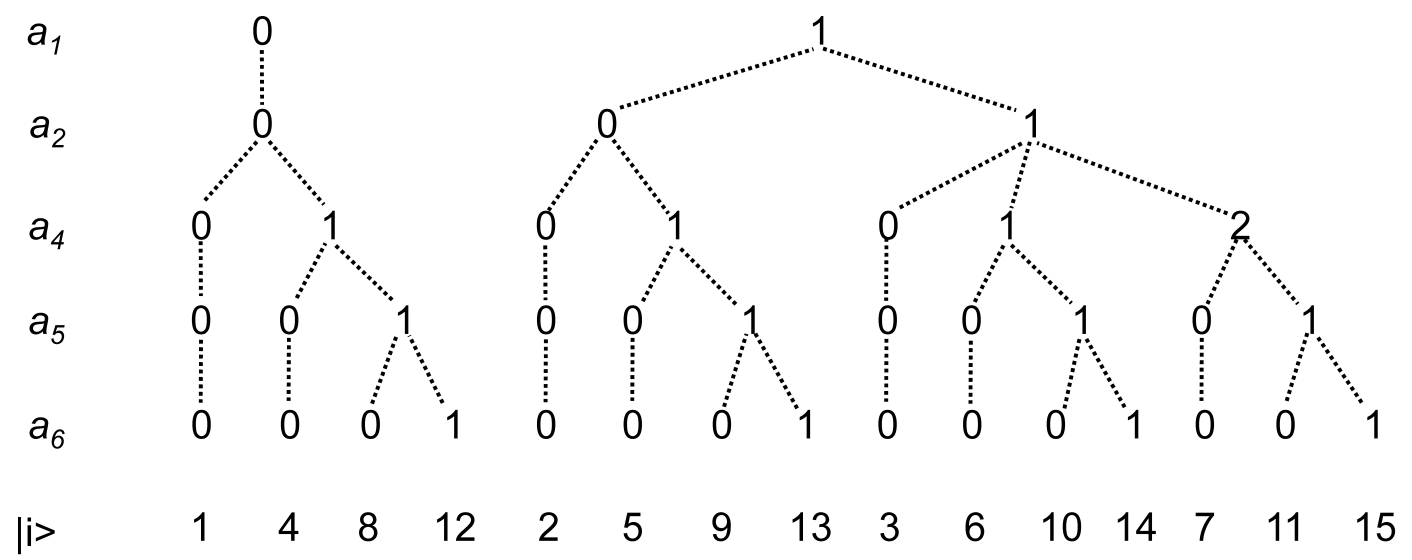

Fig. 4 List of non-vanishing exponents $a_{i}, i=1,2,4,5$ and 6 , generating Verma

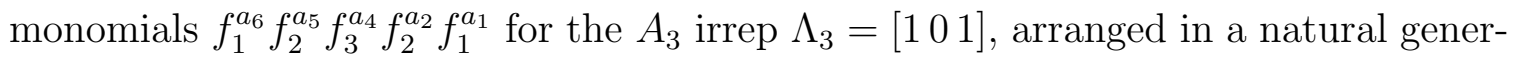
ating order. The implied lexical numbering of CSFs $|i\rangle \equiv\left|v_{i}\right\rangle, i=1, \cdots, 15$ is given in the bottom row. 


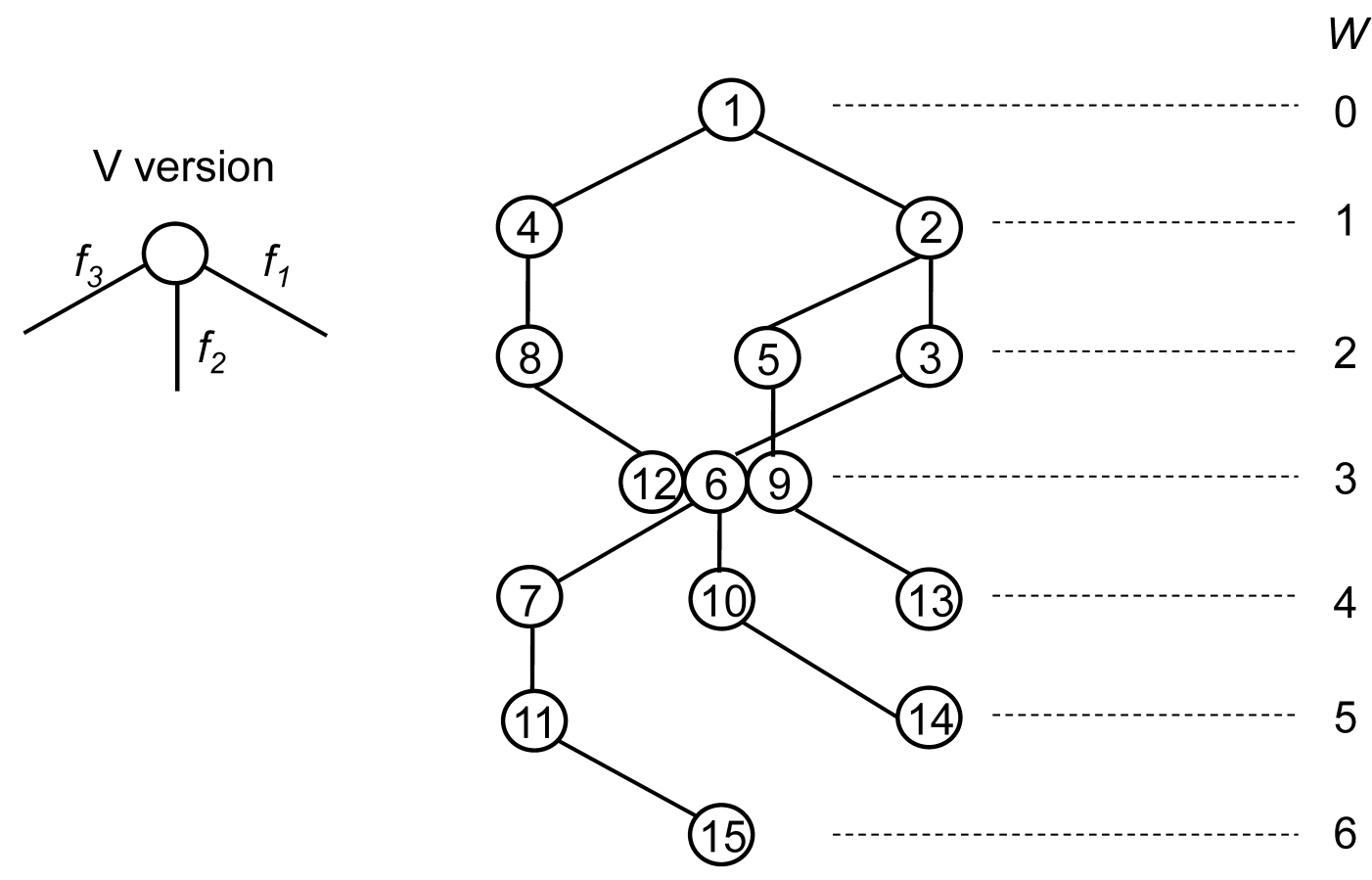

Fig. 5 The HLED diagram for the Verma basis of the U(4) irrep $\langle 2110\rangle \equiv \Gamma(121)$

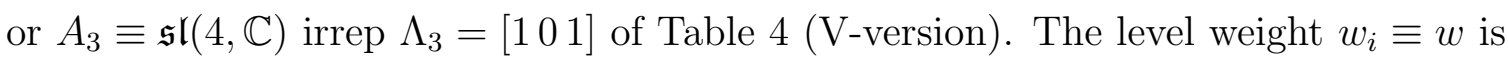
indicated on the right hand side. 


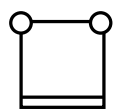

(6)

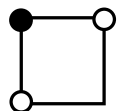

(4)

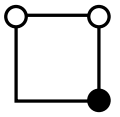

(3)

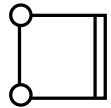

(9)

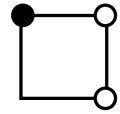

(1)

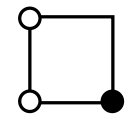

(10)

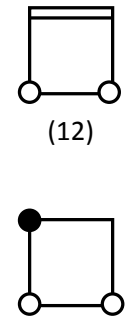

(8)

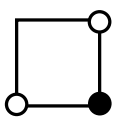

(14)

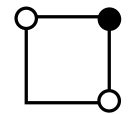

(2)

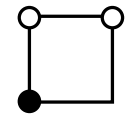

(7)

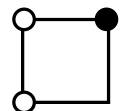

(5)

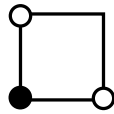

(11)

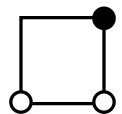

(13)

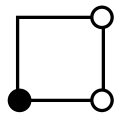

(15)

Fig. 6 VB structures representing Verma basis vectors (or CSFs) for the $\mathfrak{u}(4)$ irrep $\langle 2110\rangle \equiv \Gamma(121)$ or $A_{3}$ irrep $\Lambda_{3}=\left[\begin{array}{lll}1 & 0 & 1\end{array}\right]$ listed in the last column of Table 4, applied to the MBS PPP model of cyclobutadiene. The three valence structures are in the 1st row, the remaining rows listing singly ionic structures. No doubly-ionic structures can arise in this case. Singly occupied sites are represented by open circles and the doubly occupied ones by full circles. The numbering of the atomic sites is the same as in Fig. 2(b). 


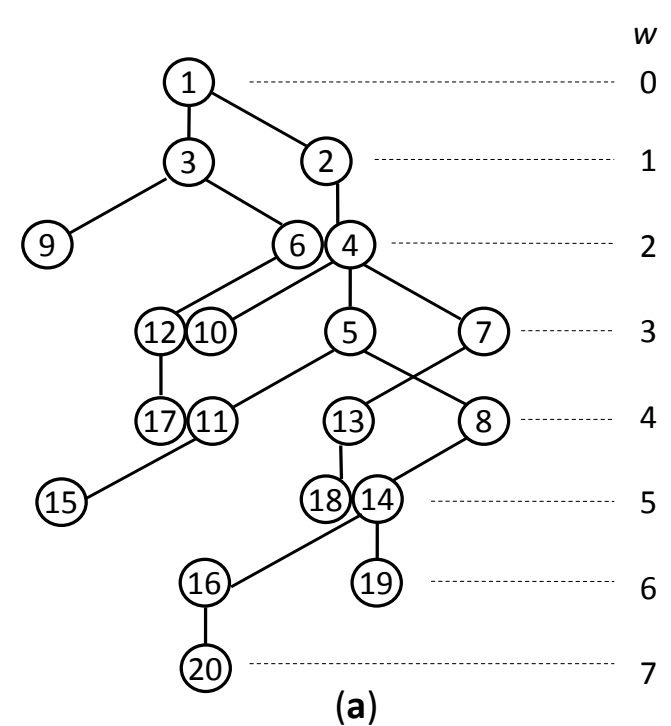

(a)

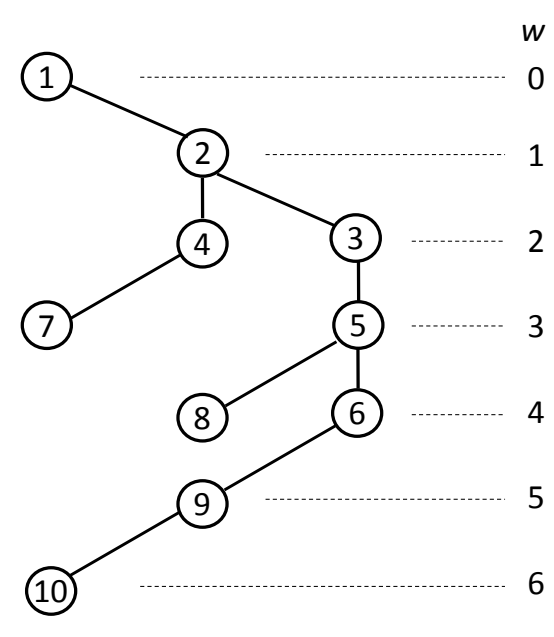

(b)

Fig. 7 The HLED diagrams for the Verma basis of the U(4) irreps (a) $\langle 2100\rangle \equiv$ $\Gamma(112)$ and $(\mathrm{b})\langle 2000\rangle\rangle \Gamma(103)$ or the $A_{3} \equiv \mathfrak{s l}(4, \mathbb{C})$ irreps (a) $\Lambda_{3}=\left[\begin{array}{lll}1 & 1 & 0\end{array}\right]$ of Table 5

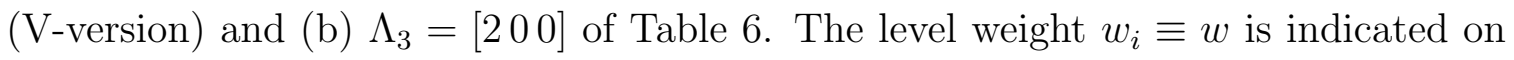
the right hand side. 


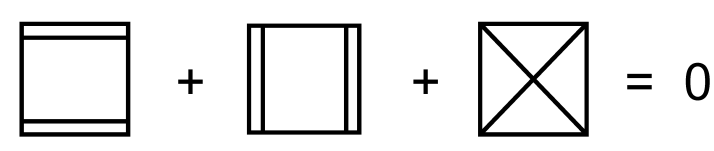

(a) (b) (c)

Fig. 8 A symbolic representation of the linear dependence of the canonical and non-canonical VB structures for the model of cyclobutadiene. 

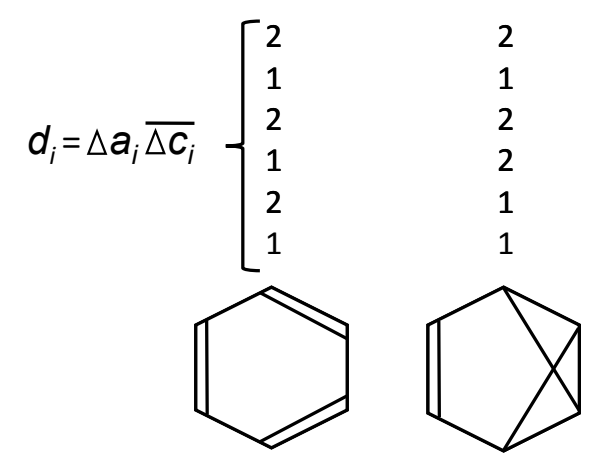

\begin{tabular}{|l|l|}
\hline 1 & 2 \\
\hline 3 & 4 \\
\hline 5 & 6 \\
\hline
\end{tabular}

\begin{tabular}{|l|l|}
\hline 1 & 3 \\
\hline 2 & 4 \\
\hline 5 & 6 \\
\hline
\end{tabular}
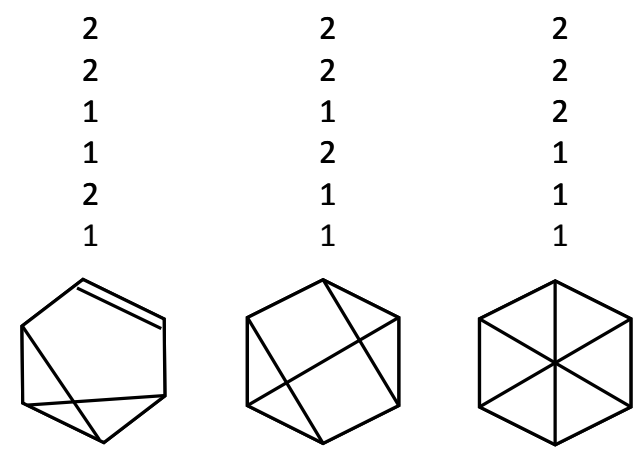

\begin{tabular}{|l|l|}
\hline 1 & 2 \\
\hline 3 & 5 \\
\hline 4 & 6 \\
\hline
\end{tabular}

\begin{tabular}{|l|l|}
\hline 1 & 3 \\
\hline 2 & 5 \\
\hline 4 & 6 \\
\hline
\end{tabular}

\begin{tabular}{|l|l|}
\hline 1 & 4 \\
\hline 2 & 5 \\
\hline 3 & 6 \\
\hline
\end{tabular}

Fig. 9 The VB structures (or Rumer patterns) that are associated with the orthonormal electronic G-T states for a singlet six-electron system (e.g., the $\pi$-electron model of the benzene molecule) corresponding to the well-known classical Kekule and Dewar structures (see also Fig. 1 and Table III of [32]). The corresponding G-T states are defined by the step-numbers $d_{i}$ given in the top row and by the Weyl tableaux in the bottom row. 
ii) $\left(a_{5} a_{4} a_{3} a_{2}\right) \quad i^{a} \equiv f_{i}^{a}$

$|5\rangle \quad\left(\begin{array}{lllll}0 & 0 & 1 & 2\end{array}\right) \quad 12^{2}$

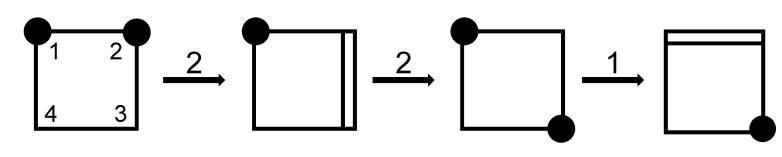

$|10\rangle \quad\left(\begin{array}{llll}0 & 1 & 1 & 2\end{array}\right) \quad 312^{2}$

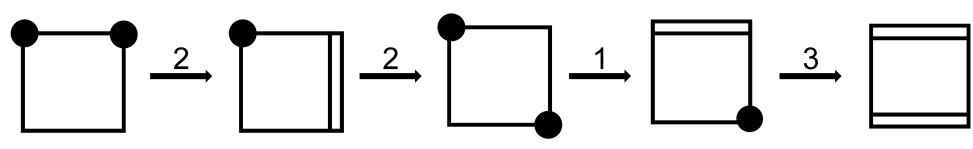

$|15\rangle$

(1 11111$)$

2312

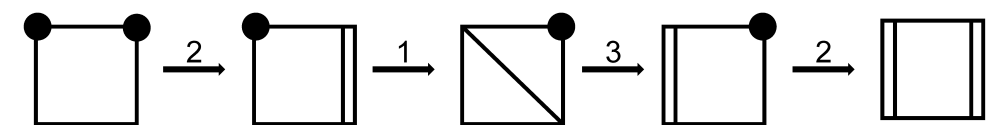

Fig. 10 Three examples of a buildup of cyclobutadiene structures for states $|5\rangle$, $|10\rangle$, and $|15\rangle$ following the relevant Verma monomials (cf. Tables 2 and 3) or the appropriate path in the HLED diagram in Fig. 3. 


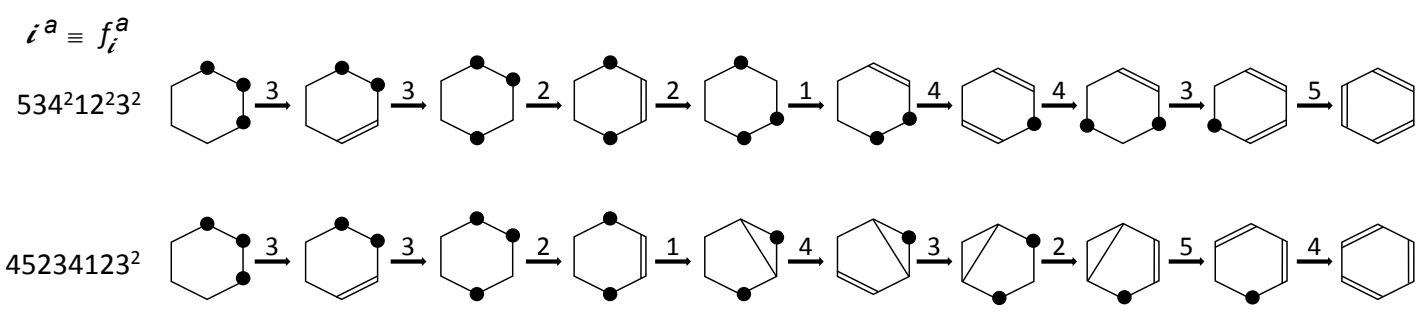

Fig. 11 A buildup of Kekulé-type covalent VB structures for the benzene model (cf. Fig. 10 for cyclobutadiene). 

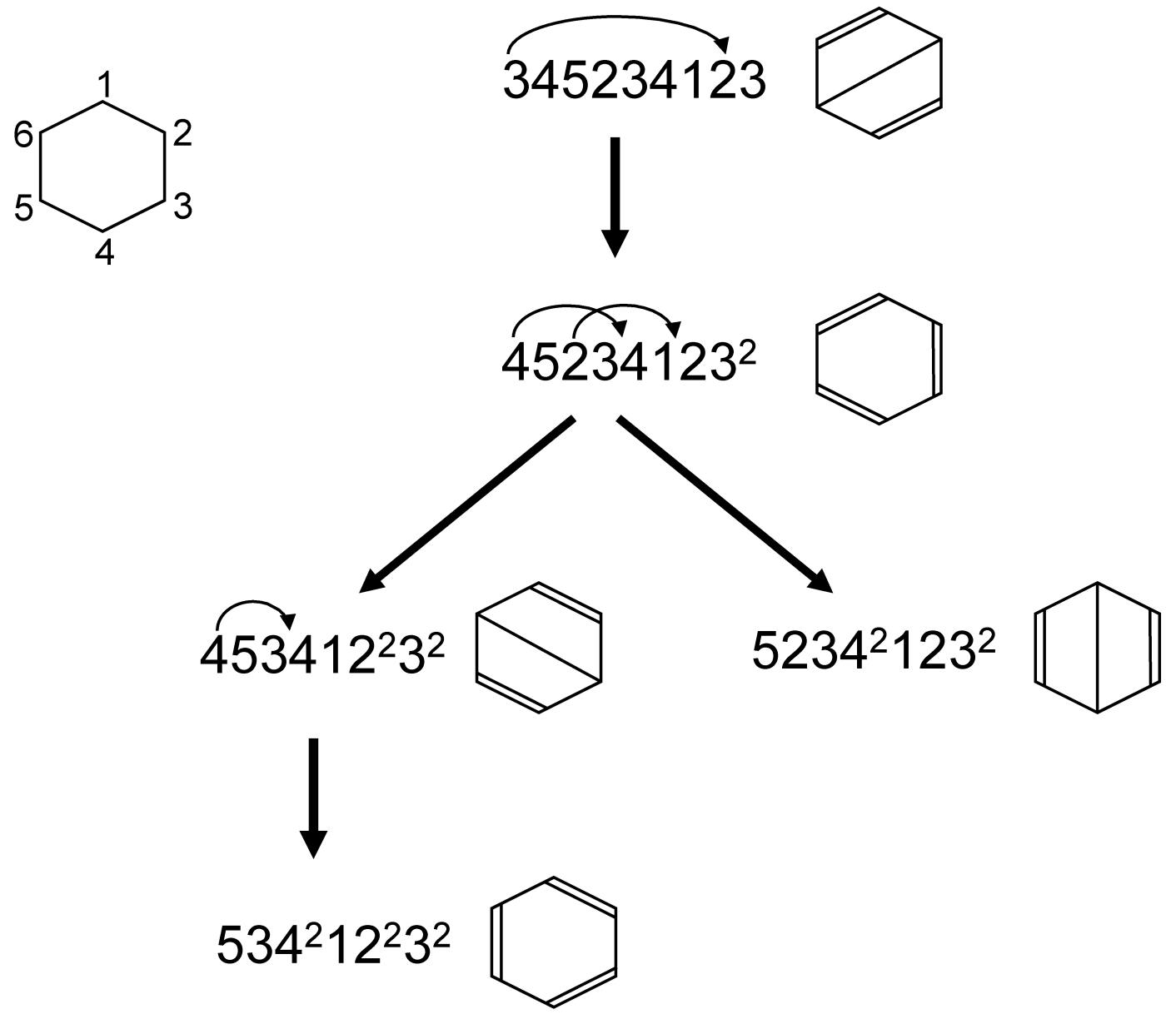

Fig. 12 An example of a generation of benzene covalent VB structures starting with the HCS (see the text for details). 


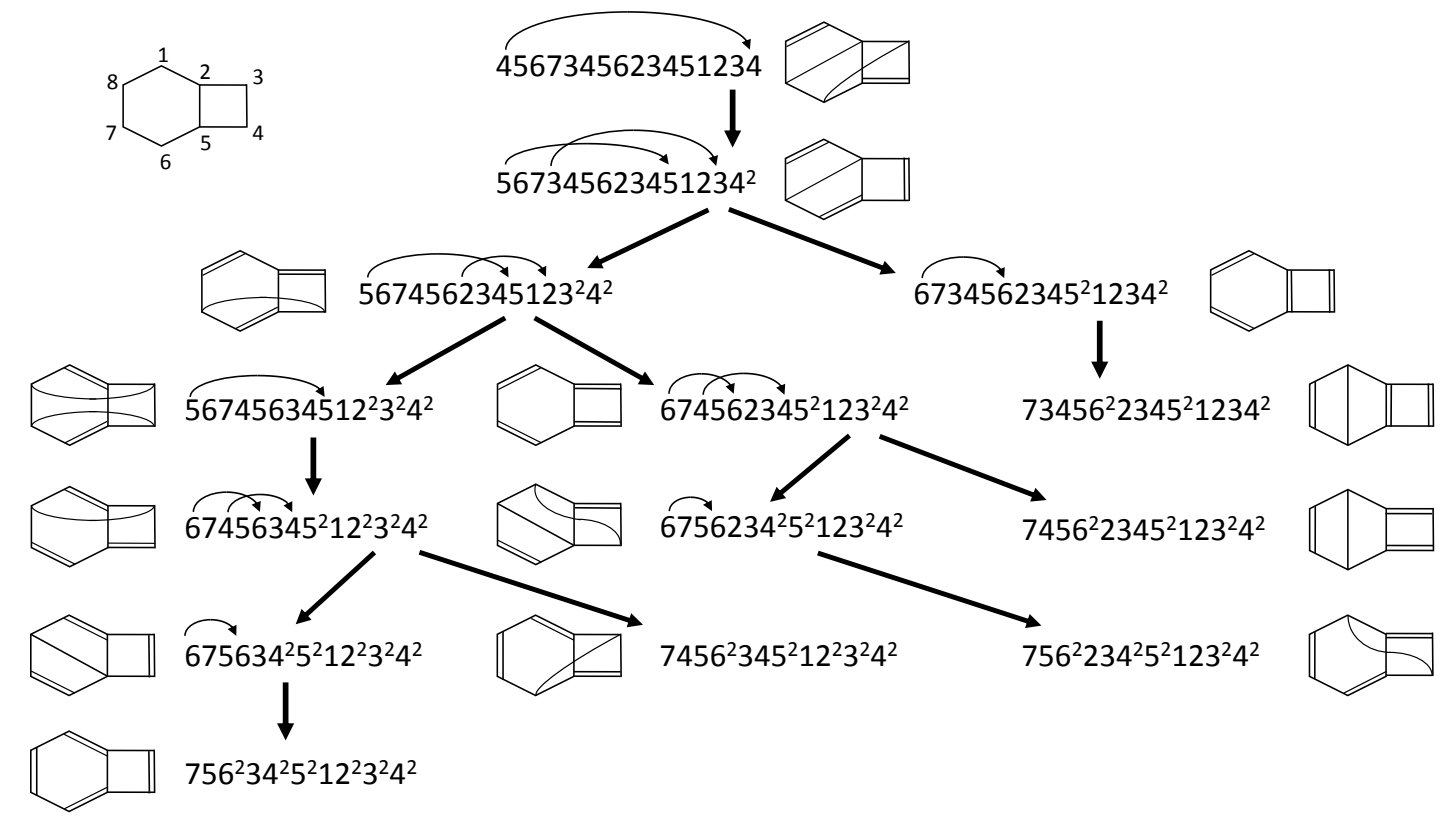

Fig. 13 An example of a generation of covalent VB structures for a 8-electron case (see the text for details). 

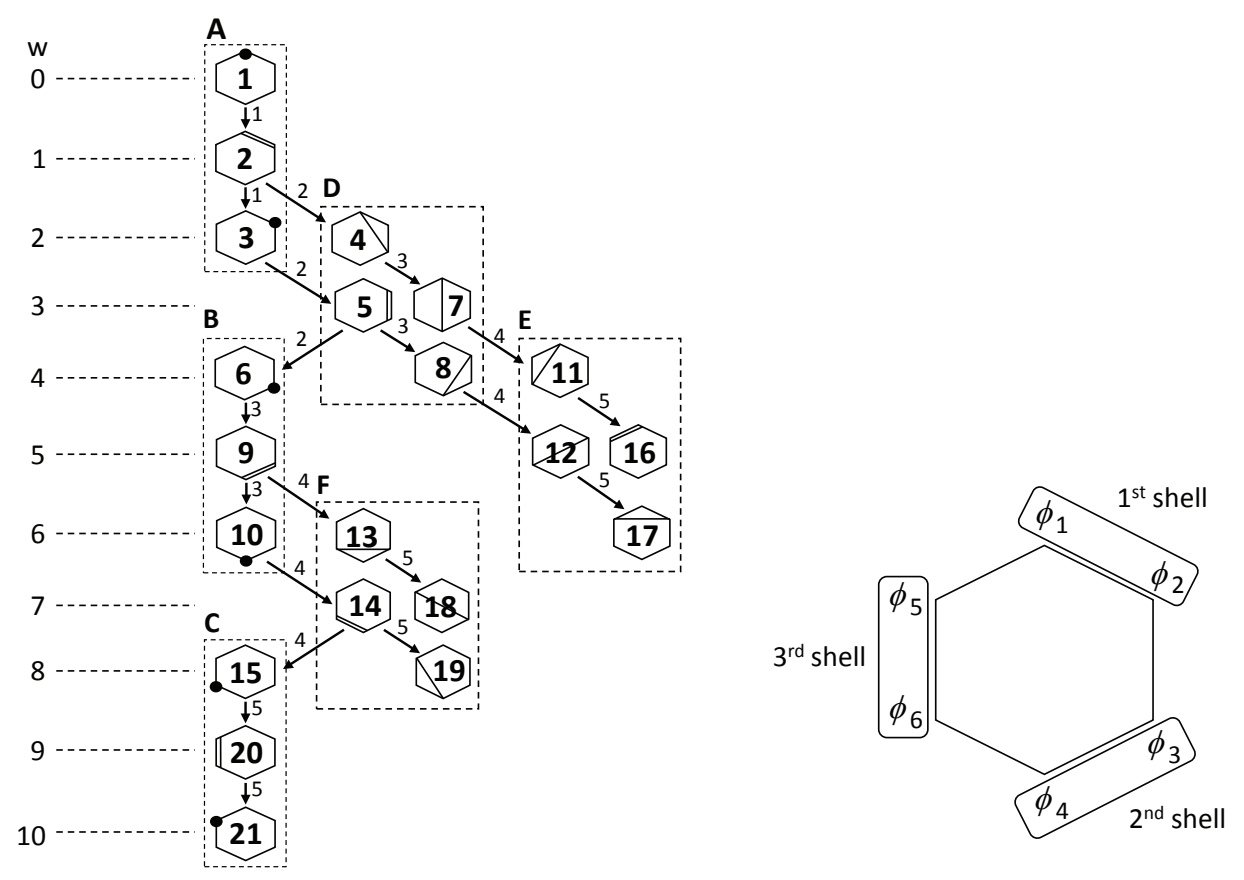

Fig. 14 HLED structure for a TZ shell-model of the ethylene or hydrogen molecules. See the text for details. 